\title{
COMPARING THE $\mathbb{Z}_{2}$-GRADED IDENTITIES OF TWO MINIMAL SUPERALGEBRAS WITH THE SAME SUPEREXPONENT
}

\author{
ONOFRIO M. DI VINCENZO AND VINCENZO NARDOZZA
}

\begin{abstract}
Let $F$ be a field of characteristic zero. We study two minimal superalgebras $A$ and $B$ having the same superexponent but such that $T_{2}(A) \varsubsetneqq$ $T_{2}(B)$, thus providing the first example of a minimal superalgebra generating a non minimal supervariety. We compare the structures and codimension sequences of $A$ and $B$.
\end{abstract}

\section{INTRODUCTION}

In PI-theory the study of the $T$-ideals of the free associative algebra has adopted the equivalent and more flexible language of varieties. The codimension sequence $\left(c_{n}(\mathscr{V})\right)_{n \in \mathbb{N}}$ of a variety $\mathscr{V}$ brought up by Regev in the seminal paper [24] is the central tool in the quantitative study of varieties, and the results [19], [20] of Giambruno and Zaicev on the exponent are among the most striking culminating points of quantitative investigations: it is possible to classify varieties on an integer scale, whose steps are the minimal varieties of given exponent. Actually, more can be said ([22]): for any $d \in \mathbb{N}$ just finitely many minimal varieties do exist, and they are generated by the Grassmann envelope of certain finite dimensional superalgebras, thus called minimal superalgebras; moreover these are exactly the varieties whose $T$-ideal is factorable as a product of verbally prime $T$-ideals (Theorem 7.5 in [22]). This last fact positively solves an early conjecture raised by Drensky [13, 14].

In the spirit of the mentioned results, varieties of algebras with additional structure have been investigated. More precisely, considering varieties of algebras with some finiteness property, it has been proved that suitable generalizations of the exponent do exist for algebras with involution ([21]) and superalgebras ([4]), but also for algebras graded by a finite group (in chronological order, $[18,2]$ and [1]). Limiting our concern to $*$-varieties and supervarieties, it has been proved that the $*$-case is very similar to the ordinary one: there are finitely many $*$-minimal $*$-varieties of fixed $*$-exponent, each generated by a suitable block-triangular matrix algebra built on $*$-simple algebras ([7]), and the converse is true as well ([9]). Perhaps surprisingly, this is unlike the case of supervarieties: any minimal supervariety is generated by a finite dimensional minimal superalgebra $([10])$, but it is still unknown which minimal superalgebras do generate minimal supervarieties.

2010 Mathematics Subject Classification. Primary 16R50, Secondary 16W50, 16W55.

Key words and phrases. Polynomial identities, superalgebra, cocharacters, codimensions, superexponent.

This is a post-print of the paper available at https://doi.org/10.1007/s10468-017-9698-8. 
Recently, a pair of minimal superalgebras was pointed out in order to give the first and simplest example of a minimal superalgebra not generating a minimal supervariety [11]. In our present paper we comparatively study these algebras: we list the generators of their superidentities, compute their graded partial codimension sequences and their module structure, and the precise rate of growth of their graded codimensions. A key role in the proofs is played by a basis of proper polynomials brought up in [8] and already proved useful in several cases. Here it provides a significant simplification in arguing the generating polynomial identities and in finding a fair linear basis for the proper multilinear spaces of the two superalgebras. Representation theory of the symmetric group, Young-derived relations for triangular algebras ([6]) and mild combinatorial arguments come further into play to complete the picture.

\section{Basic Definitions and the superalgebras $A$ And $B$}

Throughout this paper $F$ denotes a field of characteristic zero, and the word algebra means an associative, unitary $F$-algebra. A superalgebra $A=A_{0} \oplus A_{1}$ is a $\mathbb{Z}_{2}$-graded algebra. The grading is trivial when $A_{1}=0$. An ideal $I$ of a superalgebra $A$ is called homogeneous if $I=I \cap A_{0} \oplus I \cap A_{1}$. The algebra homomorphisms preserving the superalgebra structure are called graded homomorphisms. A simple superalgebra (or graded simple superalgebra) is a superalgebra having no non-trivial homogeneous ideals. Any simple algebra is graded simple; by the way, the superalgebra $D=F \oplus t F$, for $t^{2}=1$, is graded simple but not simple, and so is the matrix algebra $M_{n}(D)=M_{n}(F) \oplus t M_{n}(F)$. When dealing with matrix algebras, the customary notation $\mathbf{e}_{i j}$ denoting the matrix having $1_{F}$ as $(i, j)$-entry and $0_{F}$ elsewhere is adopted; $\mathbf{e}_{i j}$ is called the $(i, j)$-unit matrix. A convenient way to turn the full matrix algebra $M_{n}(F)$ into a superalgebra is to fix a $n$-tuple $\left(\delta_{1}, \ldots, \delta_{n}\right) \in \mathbb{Z}_{2}^{n}$ and assign to each unit matrix $\mathbf{e}_{i j}$ the $\mathbb{Z}_{2}$-degree $\delta_{j}-\delta_{i}$ (so "complementary" $n$-tuples do define the same grading). Such gradings are called the elementary gradings on $M_{n}(F)$. The free superalgebra $F\langle Y, Z\rangle$ of countable rank is the free algebra generated by the disjoint union of two infinite countable sets of letters $X=Y \cup Z$, with superalgebra structure induced by assigning degree 0 to the letters in $Y$ and degree 1 to the letters in $Z$. Since it is a free object, any graded homomorphism $\varphi: F\langle Y, Z\rangle \rightarrow A$ is uniquely defined by the images of the free letters $\varphi(x) \in A$ for all $x \in X$. Therefore $\varphi$ can be called a graded substitution. A graded polynomial identity, usually shortened in graded PI, of a superalgebra $A$ is an element of $F\langle Y, Z\rangle$ laying in the kernel of all graded substitutions in $A$. The set $T_{2}(A)$ of all graded polynomial identities of $A$ is a two-sided ideal of $F\langle Y, Z\rangle$ stable under all endomorphisms of the free algebra (a so-called $T_{2}$-ideal), and the converse is true as well, since any $T_{2}$-ideal is also graded. For any superalgebra $A$, the supervariety rising from $A$ is the class of all superalgebras $B$ such that $T_{2}(A) \subseteq T_{2}(B)$, and denoted $\mathscr{V}(A)$; in this paper, all considered supervarieties are rising from finite dimensional superalgebras. The $T_{2}$-ideal of a supervariety $\mathscr{V}$ is the intersection of the $T_{2}$-ideals of its members. Clearly, if $\mathscr{V}=\mathscr{V}(A)$ then $T_{2}(\mathscr{V})=T_{2}(A)$. Similarly, starting from a set $\mathscr{S} \subseteq F\langle Y, Z\rangle$, the supervariety generated by $\mathscr{S}$ is the class $\mathscr{V}(\mathscr{S})$ of all superalgebras $B$ whose $T_{2}$-ideal includes $\mathscr{S}$. In this case, $T_{2}(\mathscr{V})$ is the least $T_{2}$-ideal of $F\langle Y, Z\rangle$ including $\mathscr{S}$, denoted $(\mathscr{S})^{T_{2}}$, and is said generated by $\mathscr{S}$ (in particular, if a polynomial $f$ belongs to $(\mathscr{S})^{T_{2}}$ we say that $f$ follows from $\mathscr{S}$ ). 
For a given supervariety $\mathscr{V}$, the factor superalgebra $F\langle Y, Z\rangle / T_{2}(\mathscr{V})$ is a free object in the class $\mathscr{V}$, therefore is called the relatively free superalgebra of the variety.

For any $n \in \mathbb{N}$ the linear subspace

$$
\left.P_{n}^{\mathbb{Z}_{2}}:=\operatorname{span}_{F}\left\langle x_{\sigma(1)} \ldots x_{\sigma(n)}\right| \sigma \in S_{n}, x_{i} \in\left\{y_{i}, z_{i}\right\} \text { for all } i=1, \ldots, n\right\rangle
$$

is called the space of $\mathbb{Z}_{2}$-graded multilinear polynomials of degree $n$. We shall omit the superscript and simply write $P_{n}$. The whole $T_{2}$-ideal of a variety is generated by the $\mathbb{Z}_{2}$-graded multilinear polynomial it contains. For any $n \in \mathbb{N}$ the dimension

$$
c_{n}^{\mathbb{Z}_{2}}(\mathscr{V}):=\operatorname{dim} \frac{P_{n}}{P_{n} \cap T_{2}(\mathscr{V})},(n \in \mathbb{N})
$$

is called the $n$-th codimension of $\mathscr{V}$. The sequence $\left(c_{n}^{\mathbb{Z}_{2}}(\mathscr{V})\right)_{n \in \mathbb{N}}$ is the graded codimension sequence of the variety, and $\lim _{n} \sqrt[n]{c_{n}^{\mathbb{Z}_{2}(\mathscr{V})}}=: \exp ^{\mathbb{Z}_{2}}(\mathscr{V})$ is called the superexponent of the variety. This directly generalizes to supervarieties the corresponding notions given for ordinary (non graded) varieties. It has already mentioned that the limit actually exists and is a non-negative integer. Both the codimension sequence and the exponent of the varieties provide a measure on how big is $\mathscr{V}$ : clearly $\mathscr{U} \subseteq \mathscr{V}$ implies $c_{n}^{\mathbb{Z}_{2}}(\mathscr{U}) \leqslant c_{n}^{\mathbb{Z}_{2}}(\mathscr{V})$ for all $n \in \mathbb{N}$, so $\exp ^{\mathbb{Z}_{2}}(\mathscr{U}) \leqslant \exp ^{\mathbb{Z}_{2}}(\mathscr{V})$; nevertheless it may happen $\mathscr{U} \varsubsetneqq \mathscr{V}$ and yet $\mathscr{U}$ and $\mathscr{V}$ have the same exponent. Thus special consideration is deserved by those varieties whose proper subvarieties all have strictly lesser exponent, therefore called minimal supervarieties. It was already mentioned that any minimal supervariety rises from a suitable minimal superalgebra, as in the ordinary (non graded) case. The problem of selecting those minimal superalgebras actually generating a minimal supervariety is still open.

The first and easiest example has been provided in [11]: two minimal superalgebras $A$ and $B$ have been pointed out, with $T_{2}(A) \varsubsetneqq T_{2}(B)$ but giving rise to supervarieties having the same $\mathbb{Z}_{2}$-exponent. Here we recall their definition; further preliminary notions will be added in next sections, when they are needed.

Definition 1. Let $A \subseteq U T_{6}(F)$ be the 10-dimensional superalgebra whose even part $A_{0}$ is spanned by the matrices

$$
\begin{array}{ccc}
\mathbf{u}_{1}:=\mathbf{e}_{11}+\mathbf{e}_{22} & \mathbf{u}_{2}:=\mathbf{e}_{33}+\mathbf{e}_{44} & \mathbf{u}_{3}:=\mathbf{e}_{55}+\mathbf{e}_{66} \\
\mathbf{s}_{12}:=\mathbf{e}_{13}+\mathbf{e}_{24} & \mathbf{s}_{23}:=\mathbf{e}_{35}+\mathbf{e}_{46} & \mathbf{s}_{13}:=\mathbf{e}_{15}+\mathbf{e}_{26}
\end{array}
$$

and odd part $A_{1}$ is spanned by the matrices

$$
\mathbf{v}:=\mathbf{e}_{33}-\mathbf{e}_{44}, \mathbf{t}_{12}:=\mathbf{e}_{13}-\mathbf{e}_{24}, \mathbf{t}_{23}:=\mathbf{e}_{35}-\mathbf{e}_{46}, \mathbf{t}_{13}:=\mathbf{e}_{15}-\mathbf{e}_{26} .
$$

Sometimes, we shall denote $\mathbf{x}_{i j}$ an homogeneous basis element of the Jacobson radical $J(A)$, with the letter $\mathbf{x}$ to be chosen between $\mathbf{s}, \mathbf{t}$ according to the correct $\mathbb{Z}_{2}$-degree, when no immediate choice is needed, or when the choice is inessential. For instance, we may write $\mathbf{x}_{12} \mathbf{s}_{23}=\mathbf{x}_{13}$ whatever the choice of $\mathbf{x}$ is. Notice that the elected notation provides easy computation rules among the basis elements.

The linear transformation $\Theta: A \rightarrow U T_{4}(F)$ defined by

$$
\Theta:\left(\begin{array}{cccccc}
\alpha_{1} & 0 & \beta_{1} & 0 & \gamma_{1} & 0 \\
& \alpha_{1} & 0 & \beta_{2} & 0 & \gamma_{2} \\
& & \alpha_{2} & 0 & \beta_{3} & 0 \\
& & & \alpha_{3} & 0 & \beta_{4} \\
& & & & \alpha_{4} & 0 \\
& & & & & \alpha_{4}
\end{array}\right) \longrightarrow\left(\begin{array}{cccc}
\alpha_{1} & \beta_{1} & \beta_{2} & \gamma_{1}+\gamma_{2} \\
& \alpha_{2} & 0 & \beta_{3} \\
& & \alpha_{3} & \beta_{4} \\
& & & \alpha_{4}
\end{array}\right)
$$


is easily seen an algebra homomorphism, thus $B:=\Theta(A)$ is turned into a superalgebra. Let us denote $\mathbf{u}_{i}^{\prime}:=\Theta\left(\mathbf{u}_{i}\right), \mathbf{v}^{\prime}:=\Theta(\mathbf{v})$ and $\mathbf{x}_{i j}^{\prime}:=\Theta\left(\mathbf{x}_{i j}\right)$ for all $(i, j) \neq(1,3)$. Finally, set $2 \mathbf{s}_{13}^{\prime}:=\Theta\left(\mathbf{s}_{13}\right)$ and notice that $\Theta\left(\mathbf{t}_{13}\right)=\mathbf{0}$. The set $\left\{\mathbf{u}_{i}^{\prime}, \mathbf{v}^{\prime}, \mathbf{x}_{i j}^{\prime}, \mathbf{s}_{13}^{\prime}\right\}$ is clearly an homogeneous linear basis for $B$. Both $A$ and $B$ are minimal superalgebras with same graded exponent 4 and $T_{2}(A) \varsubsetneqq T_{2}(B)$, that is $\mathscr{V}(B)$ is a proper subvariety of the supervariety $\mathscr{V}(A)$ (see [11]).

\section{Graded polynomial identities of $A$ and $B$}

The easiest simple superalgebra is $F$ endowed with the trivial grading. The easiest example of graded-simple but not simple superalgebra is $D=F \oplus t F$ for some element $t$ such that $t^{2}=1$. A matrix realization of $D$ is

$$
D:=\left\{\left(\begin{array}{cc}
\alpha & \beta \\
\beta & \alpha
\end{array}\right) \mid \alpha, \beta \in F\right\}
$$

a subalgebra of $M_{2}(F)$ inheriting the $\mathbb{Z}_{2}$-elementary grading induced by the row vector $(0,1) \in \mathbb{Z}_{2}^{2}$. Thus $F$ and $D$ are the building blocks of the easiest examples of minimal superalgebras, which are the block triangular superalgebras $U T\left(\Delta_{1}, \ldots, \Delta_{k}\right)$ with $\Delta_{i} \in\{F, D\}$ for all $i=1, \ldots, k$. The algebra $A$ is actually a disguised realization of the algebra $U T(F, D, F)$ and, as such, we get the first basic result:

Theorem 2. Let $F$ be endowed with the trivial $\mathbb{Z}_{2}$-grading and let $D$ be endowed with the natural $\mathbb{Z}_{2}$-grading. Then

$$
T_{2}(A)=T_{2}(F) T_{2}(D) T_{2}(F) .
$$

Proof. Let us consider the subalgebra of $M_{5}(F)$

$$
S:=\left\{\left(\begin{array}{cc|cc|c}
a & 0 & b & c & d_{1} \\
0 & a & c & b & d_{2} \\
\hline & \alpha & \beta & d_{3} \\
& \beta & \alpha & d_{4} \\
\hline & & & & e
\end{array}\right) \mid a, b, c, d_{1}, d_{2}, d_{3}, d_{4}, e \in F\right\}
$$

inheriting the elementary grading induced by $(0,1,0,1,0) \in \mathbb{Z}_{2}^{5}$. The map defined by

$$
\left(\begin{array}{cc|cc|c}
a & 0 & b & c & d_{1} \\
0 & a & c & b & d_{2} \\
\hline & \alpha & \beta & d_{3} \\
& \beta & \alpha & d_{4} \\
\hline & & & e
\end{array}\right) \rightarrow\left(\begin{array}{cc|cc|cc}
a & 0 & b+c & 0 & d_{1}+d_{2} & 0 \\
0 & a & 0 & b-c & 0 & d_{1}-d_{2} \\
\hline & \alpha+\beta & 0 & d_{3}+d_{4} & 0 \\
& 0 & \alpha-\beta & 0 & d_{3}-d_{4} \\
\hline & & & e & 0 \\
& & & 0 & e
\end{array}\right)
$$

is easily seen to be a superalgebra isomorphism from $S$ to $A$.

Notice that $S$ is a block-triangular algebra, namely $U T\left(S_{1}, F\right)$, and $S_{1}$ is a $\mathbb{Z}_{2^{-}}$ regular subalgebra of $M_{4}(F)$, according to Definition 4.3 of [6]. Then Theorem 4.5 of [6] applies, hence

$$
T_{\mathbb{Z}_{2}}(A)=T_{\mathbb{Z}_{2}}(S)=T_{\mathbb{Z}_{2}}\left(S_{1}\right) T_{\mathbb{Z}_{2}}(F)
$$

Repeating the above procedure once again, $S_{1}$ is isomorphic to the following block-triangular subalgebra of $M_{3}(F)$ endowed with the elementary grading induced 
by $(0,0,1) \in \mathbb{Z}_{2}^{3}$

$$
S_{2}:=\left\{\left(\begin{array}{c|cc}
a & b & c \\
\hline & \alpha & \beta \\
& \beta & \alpha
\end{array}\right) \mid a, b, c, \alpha, \beta \in F\right\}
$$

via the map defined by

$$
\left(\begin{array}{c|cc}
a & b & c \\
\hline 0 & \alpha & \beta \\
0 & \beta & \alpha
\end{array}\right) \rightarrow\left(\begin{array}{cc|cc}
a & 0 & b & c \\
0 & a & c & b \\
\hline 0 & 0 & \alpha & \beta \\
0 & 0 & \beta & \alpha
\end{array}\right) .
$$

Then notice that $S_{2}$ is the block-triangular matrix algebra $U T(F, D)$ and $D$ is $\mathbb{Z}_{2}$-regular in $M_{2}(F)$, hence the same Theorem 4.5 applies once again. Therefore

$$
T_{\mathbb{Z}_{2}}\left(S_{1}\right)=T_{\mathbb{Z}_{2}}\left(S_{2}\right)=T_{\mathbb{Z}_{2}}(F) T_{\mathbb{Z}_{2}}(D)
$$

Collecting the pieces one gets $T_{2}(A)$.

Since $T_{2}(F)=\left(\left[x_{1}, x_{2}\right], z \mid x \in\{y, z\}\right)^{T_{2}}$ and $T_{2}(D)=\left(\left[x_{1}, x_{2}\right] \mid x \in\{y, z\}\right)^{T_{2}}$, the $T_{2}$-ideal of $A$ is generated by the following explicit set of polynomials

$$
\mathscr{I}:=\left\{\left[x_{1}, x_{2}\right]\left[x_{3}, x_{4}\right]\left[x_{5}, x_{6}\right], z_{1}\left[x_{2}, x_{3}\right]\left[x_{4}, x_{5}\right],\left[x_{1}, x_{2}\right]\left[x_{3}, x_{4}\right] z_{5}, z_{1}\left[x_{2}, x_{3}\right] z_{4} \mid x \in\{y, z\}\right\} .
$$

This approach provides other useful information on $A$, and we shall use them later, but very few on $B$. Therefore we are going to follow a different, more direct path providing results for both $A$ and $B$. The key point is to study the spaces of proper multilinear polynomials $\Gamma_{m, n}$ in the relatively free superalgebras of $A$ and $B$. Here we need to briefly recall some more definitions and terminology.

Let $P_{h, k}$ be the subspace of $P_{h+k}$ whose elements involve $y_{1}, \ldots, y_{h}, z_{1}, \ldots, z_{k}$ only (partial $(h, k)$-multilinear polynomials). As for $P_{n}$, any $T_{2}$-ideal is generated by the partial multilinear polynomials it contains. By the way, a further strong simplification is possible: let $[a, b]:=a b-b a$ for any $a, b \in F\langle Y, Z\rangle$. The element $[a, b]$ is called the commutator of $a, b$. Iteratively, one defines higher (left normed) commutators $\left[a_{1}, a_{2}, \ldots, a_{n}\right]=\left[\left[a_{1}, \ldots, a_{n-1}\right], a_{n}\right]$. The (unitary) subalgebra of $F\langle Y, Z\rangle$ generated by all higher commutators and the letters of $Z$ is called the algebra of $Y$-proper polynomials, denoted $B_{Y}$. Roughly speaking, a polynomial $f \in$ $F\langle Y, Z\rangle$ is in $B_{Y}$ if all the $y$ 's occur in commutators only. Then $\Gamma_{h, k}:=P_{h, k} \cap B_{Y}$. Since $1_{A}$ and $1_{B}$ are homogeneous of degree 0 , the whole $T_{2}$-ideals of $A$ and $B$ are generated by the multilinear proper polynomials they contain (see [15]). Explicitly, if $U, V \triangleleft_{T_{2}} F\langle Y, Z\rangle$, then $U=V$ if and only if $\Gamma_{h, k} \cap U=\Gamma_{h, k} \cap V$ for all $h, k \in \mathbb{N}$. We are therefore allowed to work with much smaller multilinear spaces. In this paper we are in particular interested in a basis of $B_{Y}$ described in [8], which is especially useful when a $T_{2}$-ideal is generated by products of commutators: let us fix a total order in $X=Y \cup Z$ such that $Y<Z$; a commutator $c=\left[x_{1}, x_{2}, \ldots, x_{k}\right]$ is a called a normal standard higher commutator (shortened in nshc) if either $c=1_{F}$ (commutator of length 0 ), either $c=z \in Z$ (commutator of length 1 ) or in the general case

$$
c=\left[x, y_{i_{1}}, y_{i_{2}}, \ldots, y_{i_{k}}\right] \text { where } x>y_{i_{1}}<y_{i_{2}}<\cdots<y_{i_{k}} \text { and } k \geqslant 1,
$$

a regular commutator of length $k$. The products of nshc's in the letters $y_{1}, \ldots, y_{m}$, $z_{1}, \ldots, z_{n}$ form the basis for $\Gamma_{m, n}$ obtained in [8].

When nor the precise knowledge of the letters $y_{i_{1}}, \ldots, y_{i_{k}}$, nor the precise value of $k \geqslant 1$ is needed, we shall write simply $c_{i}=\left[x_{i}, Y_{(i)}\right]$ to denote the $i$-th regular 
commutator (that is, of length $\geqslant 2$ ) occurring in a product of nshc's. Similarly, if in the product $z_{a} z_{i_{1}} z_{i_{2}} \ldots z_{i_{k}} z_{b}$ the word $z_{i_{1}} z_{i_{2}} \ldots z_{i_{k}}$ is standard, that is $z_{i_{1}}<$ $z_{i_{2}}<\cdots<z_{i_{k}}$, and there is no need of knowing the precise letters occurring in it, we shall simply write $z_{a} w z_{b}$; hence $w$ will always denote a standard word in some suitable letters from $Z$, possibly the empty word.

We are now interested in finding a basis for the proper multilinear spaces $\Gamma_{m, n}(A)=$ $\Gamma_{m, n} /\left(\Gamma_{m, n} \cap T_{2}(A)\right)$ and $\Gamma_{m, n}(B)=\Gamma_{m, n} /\left(\Gamma_{m, n} \cap T_{2}(B)\right)$. These bases will be central in almost the whole paper; in this section, we shall exploit them to exhibit a generating set for $T_{2}(B)$.

Definition 3. Let us consider the following classes of proper polynomials:

- For $m \geqslant 2$ and $n \geqslant 0$ set $d\left(x_{1}, Y_{(1)}, x_{2}\right):=\left[x_{1}, Y_{(1)}\right] w\left[x_{2}, Y_{(2)}\right] \in \Gamma_{m, n}$. Notice that

- $y_{1}$ (the least letter in $Y$ ) occurs always as first letter in $Y_{(1)}$ or $Y_{(2)}$;

$-w$ may be empty (but then $n<3$ );

$-d\left(x_{1}, Y_{(1)}, x_{2}\right)$ is uniquely determined by $x_{1}, Y_{(1)}, x_{2}$ and $(m, n)$.

- For $m \geqslant 1$ and $n \geqslant 1$ (but $m+n \geqslant 3$ ) set $s(z, x):=z w\left[x, Y_{(1)}\right]$. Notice that:

$-w$ may be empty;

$-y_{1}$ must be the first letter of $Y_{(1)}$;

$-s(z, x)$ is uniquely determined by $z, x$ and $(m, n)$

- For $m \geqslant 1, n \geqslant 1$ and $m+n \geqslant 3$ set $\bar{s}(z, x):=\left[x, Y_{(1)}\right] w z$, where $s(z, x)=$ $z w\left[x, Y_{1}\right]$.

- If $n \leqslant 1$ (but $m+n \geqslant 2$ ) set $c(x):=\left[x, Y_{(1)}\right]$, a single nshc of length $\geqslant 2$.

- If $m=0$ and $n \geqslant 2$ set $p(z, \bar{z}):=z w \bar{z}$. Of course, $w$ may possibly be the empty word.

Let $\mathscr{G}$ be the set of all those polynomials, together with all multilinear proper polynomials of total degree $\leqslant 3$. We write $\mathscr{G}_{m, n}=\mathscr{G} \cap \Gamma_{m, n}$.

Proposition 4. For all $m, n \in \mathbb{N}$ the set $\mathscr{G}_{m, n}$ spans $\Gamma_{m, n}$ modulo $T_{2}(A)$.

Proof. If $m+n \leqslant 3$ then all the standard basis elements of $\Gamma_{m, n}$ are in $\mathscr{G}$, so assume $m+n \geqslant 4$, and let $f$ be a basis element of $\Gamma_{m, n}$. We are going to prove that $f$ can be written as a linear combination of elements of $\mathscr{G}_{m, n}$ modulo $T_{2}(A)$. Let $k$ be the number of regular commutators occurring in $f$. Using the basic commutation rule $b a=a b+[b, a]$, it is easy to see that if $k \geqslant 3$ then $f$ is a consequence of $\left[x_{1}, x_{2}\right]\left[x_{3}, x_{4}\right]\left[x_{5}, x_{6}\right] \in \mathscr{I}$, therefore $f \equiv 0 \bmod T_{2}(A)$. If $k=2$, then $f=u_{1} c_{1} u_{2} c_{2} u_{3}$, for suitable words $u_{1}, u_{2}, u_{3}$ in letters from $Z$ only; as before, it is easy to see that if at least one among $u_{1}, u_{3}$ is not the empty word then $f \equiv 0 \bmod T_{2}(A)$ because of the identity $z\left[x_{1}, x_{2}\right]\left[x_{3}, x_{4}\right]$ or $\left[x_{1}, x_{2}\right]\left[x_{3}, x_{4}\right] z$. So if $k=2$ and $f \not \equiv 0 \bmod T_{2}(A)$ then $u_{1} u_{3}=1$. Denote with $l(u)$ the length of a word $u$ (that is, the total degree of $u$ ). If $l\left(u_{2}\right)<2$ then $u_{2}$ is standard, and $f=d\left(x_{1}, Y_{1}, x_{2}\right) \in \mathscr{G}$. So assume $l\left(u_{2}\right) \geqslant 2$. If $u_{2}$ is already standard, we are done; otherwise we may reduce $u_{2}$ to a standard word working modulo $T_{2}(A)$ : let $u_{2}=u^{\prime} \bar{z} z u^{\prime \prime} \operatorname{con} \bar{z}>z$. Then

$$
c_{1} u_{2} c_{2}=c_{1} u^{\prime} z \bar{z} u^{\prime \prime} c_{2}+c_{1} u^{\prime}[\bar{z}, z] u^{\prime \prime} c_{2} \equiv c_{1} u^{\prime} z \bar{z} u^{\prime \prime} c_{2} \quad \bmod T_{2}(A)
$$

and the word $u^{\prime} z \bar{z} u^{\prime \prime}$ has strictly less inversions than the word $u_{2}$. Iteratively, one gets $f \equiv c_{1} w c_{2} \bmod T_{2}(A)$. Notice that $w$ is standard on the same set of letters occurring in $u_{2}$. 
Now assume $k=1$, say $f=z u_{1}\left[x, Y_{(1)}\right] u_{2}$ or $f=u_{1}\left[x, Y_{(1)}\right] u_{2} z$, for suitable (possibly empty) words $u_{1}, u_{2} \in F\langle Z\rangle$. Of course, if $u_{1}=u_{2}=1_{F}$ then $f=s(z, x)$ or $f=\bar{s}(z, x)$; on the other edge, if both $u_{1}, u_{2}$ are not empty then $f \equiv 0(\bmod I)$ because $f$ follows from $z_{1}\left[x_{2}, x_{3}\right] z_{2} \in \mathscr{I}$. Finally, assume $u_{1} \neq 1$ and $f \not \equiv 0$ $\bmod T_{2}(A)$. This implies $u_{2}=1$ (and $f=z u_{1}\left[x, Y_{(1)}\right]$ ), and it is possible to straighten $u_{1}$ getting a standard word: if $u_{1}=u^{\prime} \overline{\bar{z}} \bar{z} u^{\prime \prime}$ with $\overline{\bar{z}}>\bar{z}$ then

$$
z u_{1} c=z u^{\prime} \bar{z} \overline{\bar{z}} u^{\prime \prime} c+z u^{\prime}[\overline{\bar{z}}, \bar{z}] u^{\prime \prime} c \equiv z u^{\prime} \bar{z} \overline{\bar{z}} u^{\prime \prime} c \quad \bmod T_{2}(A)
$$

and iterativaly one gets $f \equiv z w\left[x, Y_{(1)}\right]=s(z, x) \bmod T_{2}(A)$. Clearly the case $u_{1}=1, u_{2} \neq 1$ and $f \not \equiv 0 \bmod T_{2}(A)$ is similar.

Finally, if $k=0$ then $f$ is a monomial in letters from $Z$ only. Since $m+n=n \geqslant 4$ we may write $f=z u \bar{z}$ and $l(u) \geqslant 2$. The word $u$ can be straightened thanks to the identity $z_{1}\left[x_{2}, x_{3}\right] z_{4} \in \mathscr{I}$, thus getting $f \equiv z w \bar{z}=p(z, \bar{z}) \bmod T_{2}(A)$.

What is left to do is to show that for any $m, n \in \mathbb{N}$ the set $\mathscr{G}_{m, n}(A):=\{f+$ $\left.\left(\Gamma_{m, n} \cap T_{2}(A)\right) \mid f \in \mathscr{G}_{m, n}\right\}$ is actually an $F$-basis of $\Gamma_{m, n}(A)$. We shall commit a harmless abuse of notation writing simply $f$ instead of $f+\left(\Gamma_{m, n} \cap T_{2}(A)\right)$ to denote an element of the factor space when no confusion may arise.

Notice that if $m+n \leqslant 3$ then $\mathscr{G}_{m, n}$ is trivially linearly independent, since $\Gamma_{m, n} \cap$ $T_{2}(A)=0$; hence we assume $m+n \geqslant 4$. We separate few cases in different lemmas.

Lemma 5. For all $m \geqslant 4$ the set $\mathscr{G}_{m, 0}(A)$ is a basis for $\Gamma_{m, 0}(A)$.

Proof. If $\mathscr{G}_{m, n}$ is not linearly independent modulo $T_{2}(A)$ then there exists a minimal subset $\mathscr{S} \subseteq \mathscr{G}_{m, n}$ such that $\sum_{b \in \mathscr{S}} \alpha_{b} b \equiv 0 \bmod T_{2}(A)$ for some scalars $\alpha_{b}$ all nonzero.

Now choose any $c(y)=\left[y, Y_{(1)}\right] \in \mathscr{G}_{m, 0}$. The graded substitution

$$
\varphi:=\begin{array}{ll}
y & Y_{(1)} \\
\mathbf{s}_{12} & \mathbf{u}_{2}
\end{array}
$$

sending $y \rightarrow \mathbf{s}_{12}$ and all letters of $Y_{(1)}$ in $\mathbf{u}_{2}$, does not vanish on $c(y)$ while vanishes on all other elements on $\mathscr{G}_{m, 0}$ : it is $\varphi(c(y))=\mathbf{s}_{12}$, and for all $d=d\left(y^{\prime}, Y_{(1)}^{\prime}, \bar{y}^{\prime}\right) \in$ $\mathscr{G}_{m, 0}$ it is clearly $\varphi(d)=\mathbf{0}$. Next, if $\mathscr{G}_{m, 0} \ni c\left(y^{\prime}\right)=\left[y^{\prime}, Y_{(1)}^{\prime}\right] \neq c(y)$ then $y^{\prime} \neq y$ and $y^{\prime}>y_{1}$, which is inevitably the first letter of $Y_{(1)}^{\prime}$. Therefore $\varphi\left(c\left(y^{\prime}\right)\right)=$ $\left[\mathbf{u}_{2}, \mathbf{u}_{2}, \ldots\right]=\mathbf{0}$.

For this reason, none of the $b$ 's occurring in $\sum_{b} \alpha_{b} b$ can be of type $c(y)$. Hence all of them are of type $d$.

By the way, choose any $d=d\left(y, Y_{(1)}, \bar{y}\right)=\left[y, Y_{(1)}\right]\left[\bar{y}, Y_{(2)}\right]$; we then may exhibit a graded substitution vanishing on all $d^{\prime} \in \mathscr{G}_{m, 0}$ but the chosen one:

$$
\varphi:=\begin{array}{llll}
y & Y_{(1)} & \bar{y} & Y_{(2)} \\
\mathbf{s}_{12} & \mathbf{u}_{1} & \mathbf{s}_{23} & \mathbf{u}_{3}
\end{array}
$$

Up to the sign, it is $\varphi(d)=\mathbf{s}_{13}$. Now let $d^{\prime}=d\left(y^{\prime}, Y_{(1)}^{\prime}, \bar{y}^{\prime}\right)=\left[y^{\prime}, Y_{(1)}^{\prime}\right]\left[\bar{y}^{\prime}, Y_{(2)}^{\prime}\right] \in$ $\mathscr{G}_{m, 0}$ and assume $\varphi\left(d^{\prime}\right) \neq \mathbf{0}$. We want to prove that $d^{\prime}=d$.

Of course $\varphi\left(d^{\prime}\right)$ is an even element of the Jacobson radical of $A$, more precisely $\varphi\left(d^{\prime}\right) \in J_{0}^{2}=F \mathbf{s}_{13}$. Therefore $\varphi\left(d^{\prime}\right) \neq \mathbf{0}$ implies $\varphi\left(\left[y^{\prime}, Y_{1}^{\prime}\right]\right)=\mathbf{s}_{12}$ and $\varphi\left(\left[\bar{y}^{\prime}, Y_{2}^{\prime}\right]\right)=$ $\mathbf{s}_{23}$. Hence $y$ must occur in $c_{1}^{\prime}$ in position 1 or 2 and $\bar{y}$ must occur in $c_{2}^{\prime}$ in position 1 or 2. On the other hand, $y_{1}=\min Y_{(1)} \cup Y_{(2)}$ must be the first element of $Y_{(1)}^{\prime}$ or $Y_{(2)}^{\prime}$, but is easy to see that if $y_{1} \in Y_{2}^{\prime}$ then $c_{2}^{\prime}=\left[\bar{y}, y_{1}, \ldots\right]$ and $\varphi\left(c_{2}^{\prime}\right)=\left[\mathbf{s}_{23}, \mathbf{u}_{1}, \ldots\right]=\mathbf{0}$. 
Thus $y_{1}$ must occur in $Y_{1}^{\prime}$ so $y^{\prime}=y$. Moreover it must be $Y_{1}^{\prime} \subseteq Y_{1}$, since $\left[\mathbf{s}_{12}, \mathbf{u}_{3}\right]=\mathbf{0}$. Let us set $y_{0}:=\min Y_{(2)}^{\prime}$. It must happen $\bar{y}^{\prime}, y_{0} \notin Y_{1}: \bar{y}$ must be one of them, hence $\varphi\left(\left[\bar{y}^{\prime}, y_{0}\right]\right)= \pm\left[\mathbf{u}_{1}, \mathbf{s}_{23}\right]=\mathbf{0}$. Moreover, if $\exists y_{k} \in Y_{1} \backslash Y_{1}^{\prime}$, the letter $y_{k}$ should occur in $Y_{(2)}^{\prime}$ and $y_{k}>y_{0}$, therefore $\varphi\left(\left[\bar{y}^{\prime}, Y_{2}^{\prime}\right]\right)= \pm\left[\mathbf{s}_{23}, \mathbf{u}_{3}, \ldots, \mathbf{u}_{1}, \ldots\right]=\mathbf{0}$, a contradiction. Hence $Y_{1}^{\prime}=Y_{1}$, so $c_{1}=c_{1}^{\prime}$. This implies $\{\bar{y}\} \cup Y_{(2)}=\left\{\bar{y}^{\prime}\right\} \cup Y_{(2)}^{\prime}$ (because $d$ and $d^{\prime}$ have the same letters). In particular these sets have the same minimum, and it must be $y_{0}: \min \left(\{\bar{y}\} \cup Y_{(2)}\right)=\min Y_{(2)}$ because $\bar{y}>\min Y_{(2)}$, and all the same $\min \left(\left\{\bar{y}^{\prime}\right\} \cup Y_{(2)}^{\prime}\right)=\min Y_{(2)}^{\prime}=y_{0}$. Therefore $\min Y_{(2)}=y_{0}$ and it occurs in position 2 both in $c_{2}$ as in $c_{2}^{\prime}$. This implies $\bar{y}$ is in position 1 in $c_{2}^{\prime}$ as well, so $c_{2}^{\prime}=c_{2}$.

For these reasons, the only linear combination of polynomials of type $d$ lying in $T_{2}(A)$ is the trivial one, that is $\mathscr{S}=\varnothing$.

The proofs of the next results are very similar to the previous one, and we will just give the essential details.

Lemma 6. For all $m \geqslant 3$ the set $\mathscr{G}_{m, 1}(A)$ is a basis for $\Gamma_{m, 1}(A)$.

Proof. Let $\mathscr{S} \subseteq \mathscr{G}_{m, 1}$ be a minimal linearly dependent set $\bmod T_{2}(A)$ and assume $\sum_{b \in \mathscr{S}} \alpha_{b} b \equiv 0 \bmod T_{2}(A)$. The only polynomial $c=\left[z, Y_{(1)}\right] \in \mathscr{G}_{m, 1}$ cannot be in $\mathscr{S}$ because the substitution $z \rightarrow \mathbf{t}_{12}, Y_{(1)} \rightarrow \mathbf{u}_{2}$ sends to $\mathbf{0}$ all polynomials of type $d, s$ and $\bar{s}$ while does not vanish on $c$.

If $s=z\left[y, Y_{(1)}\right]$ the substitution

$$
\varphi: \begin{array}{lll}
z & y & Y_{(1)} \\
\mathbf{v} & \mathbf{s}_{23} & \mathbf{u}_{3}
\end{array}
$$

satisfies $\varphi(s) \neq \mathbf{0}$ while vanishes on all $s^{\prime} \neq s$, and on all polynomials of type $\bar{s}, d$. A similar reasoning works starting from a polynomial $\bar{s}(z, y)$ (this time the substitution is $y \rightarrow \mathbf{s}_{12}, Y_{(1)} \rightarrow \mathbf{u}_{1}$ e $z \rightarrow \mathbf{v}$ ), so just polynomials of type $d$ could possibly be in $\mathscr{S}$.

Take at first any $d=\left[z, Y_{(1)}\right]\left[y, Y_{(2)}\right]$ and consider the substitution

$$
\varphi: \begin{array}{llll}
z & Y_{(1)} & y & Y_{(2)} \\
\mathbf{t}_{12} & \mathbf{u}_{1} & \mathbf{s}_{23} & \mathbf{u}_{3}
\end{array}
$$

Then $\varphi\left(d^{\prime}\right)=\mathbf{0}$ for all polynomials $d^{\prime} \neq d$, while $\varphi(d) \neq \mathbf{0}$. Essentially the same considerations hold starting from $d=\left[y, Y_{1}\right]\left[z, Y_{2}\right]$.

Hence just polynomials $d=\left[y_{1}, Y_{(1)}\right] z\left[y_{2}, Y_{(2)}\right]$ could possibly be in $\mathscr{S}$. For a fixed $d$, the substitution

$$
\varphi=\begin{array}{lllll}
y_{1} & Y_{(1)} & z & y_{2} & Y_{(2)} \\
\mathbf{s}_{12} & \mathbf{u}_{1} & \mathbf{v} & \mathbf{s}_{23} & \mathbf{u}_{3}
\end{array}
$$

satisfies $\varphi(d) \neq \mathbf{0}$, but for all $d^{\prime}=\left[x_{1}, Y_{1}^{\prime}\right] z\left[x_{2}, Y_{2}^{\prime}\right] \neq d$ it is $\varphi\left(d^{\prime}\right)=\mathbf{0}$. Therefore $\mathscr{S}=\varnothing$ and $\mathscr{G}_{m, 1}$ is linearly independent modulo $T_{2}(A)$.

Lemma 7. For all $m, n \geqslant 2$ the set $\mathscr{G}_{m, n}(A)$ is a basis for $\Gamma_{m, n}(A)$.

Proof. Let $\mathscr{S} \subseteq \mathscr{G}_{m, n}$ be a minimal linearly dependent set modulo $T_{2}(A)$, and assume $\sum_{b \in \mathscr{S}} \alpha_{b} b \equiv 0 \bmod T_{2}(A)$. In principle, just polynomials of type $d, s$ and $\bar{s}$ can be in $\mathscr{S}$. By the way, if $s(z, x)=z w\left[x, Y_{(1)}\right]$ (with possibly $w=1$ ) the substitution

$$
\varphi: \begin{array}{llll}
z & w & x & Y_{(1)} \\
\mathbf{t}_{12} & \mathbf{v} & \mathbf{x}_{23} & \mathbf{u}_{3}
\end{array}
$$


$\left(\mathbf{x} \in\{\mathbf{s}, \mathbf{t}\}\right.$ according to the $\mathbb{Z}_{2}$-degree of $\left.x\right)$ does not vanish on $s$ while is zero on all other $b$. Similar considerations hold for any $\bar{s}(z, x)$. Hence just polynomials of type $d$ may be in $\mathscr{S}$.

We have to consider the special case $n=2$ : fix $d=\left[z_{1}, Y_{(1)}\right]\left[z_{2}, Y_{(2)}\right]$. Then the needed substitution is

$$
\varphi=\begin{array}{llll}
z_{1} & Y_{(1)} & z_{2} & Y_{(2)} \\
\mathbf{t}_{12} & \mathbf{u}_{1} & \mathbf{t}_{23} & \mathbf{u}_{3}
\end{array} .
$$

Thus just polynomials $d=\left[x_{1}, Y_{1}\right] w\left[x_{2}, Y_{2}\right]$, where $w \neq 1$, could possibly be in $\mathscr{S}$, and we can deal with the unique general case $n \geqslant 2$.

If $d=\left[x_{1}, Y_{1}\right] w\left[x_{2}, Y_{2}\right]$ we point out the substitution

$$
\varphi=\begin{array}{lllll}
x_{1} & Y_{1} & w & x_{2} & Y_{2} \\
\mathbf{x}_{12} & \mathbf{u}_{1} & \mathbf{v} & \mathbf{x}_{23} & \mathbf{u}_{3}
\end{array}
$$

sending to zero all $d^{\prime}$ but $d$, so $\mathscr{S}=\varnothing$.

The last two cases to complete the description are

Lemma 8. For all $n \geqslant 3$ the set $\mathscr{G}_{1, n}(A)$ is a basis for $\Gamma_{1, n}(A)$

and

Lemma 9. For all $n \geqslant 4$ the set $\mathscr{G}_{0, n}(A)$ is a basis for $\Gamma_{0, n}(A)$.

The proofs of these results follow as particular cases of previous ones (the former) or are immediate (the latter).

In order to get a generating set for $T_{2}(B)$ we need to add other identities to those in $\mathscr{I}$. The natural attempt is to try with the polynomials $\left[x_{1}, x_{2}\right]\left[x_{3}, x_{4}\right]$ having $\mathbb{Z}_{2}$-degree 1 , that is the polynomials in

$$
\mathscr{C}:=\left\{\left[z_{1}, y_{2}\right]\left[y_{3}, y_{4}\right],\left[y_{1}, y_{2}\right]\left[z_{3}, y_{4}\right],\left[z_{1}, z_{2}\right]\left[z_{3}, y_{4}\right],\left[z_{1}, y_{2}\right]\left[z_{3}, z_{4}\right]\right\} .
$$

It is indeed straightforward to see that $\mathscr{C} \subseteq T_{2}(B)$. One easily gets

Lemma 10. Let $f=\left[x_{1}, x_{2}\right] u\left[x_{3}, x_{4}\right]$ where $u$ is a monomial in $F\langle Z\rangle$. If $f$ has $\mathbb{Z}_{2}$-degree 1 then $f$ follows from $\mathscr{C}$ modulo $T_{2}(A)$. In particular, $f \in T_{2}(B)$.

Plainly any regular commutator $\left[x, Y_{(1)}\right]$ is a consequence of $\left[x, y_{1}\right]$. Then, denoting from now on $\partial(f)$ the $\mathbb{Z}_{2}$-degree of a polynomial $f \in F\langle Y, Z\rangle$, from the previous Lemma it follows

Corollary 11. If $d=d\left(x_{1}, Y_{(1)}, x_{2}\right)$ has $\partial(d)=1$ then $d$ follows from $\mathscr{C}$ modulo $T_{2}(A)$. In particular, $d \in T_{2}(B)$.

Let $U$ be the $T_{2}$-ideal generated by the set $\mathscr{I} \cup \mathscr{C}$. Then $T_{2}(A) \subseteq U \subseteq T_{2}(B)$, and we want to prove $U=T_{2}(B)$. This will be achieved if we prove that $\Gamma_{m, n} \cap U=$ $\Gamma_{m, n} \cap T_{2}(B)$ for all $m, n \in \mathbb{N}$, and this it true if and only if we succede in finding for all $m, n \in \mathbb{N}$ a set of proper polynomials spanning $\Gamma_{m, n}$ modulo $U$ and linearly independent modulo $T_{2}(B)$.

About the first request, certainly $\mathscr{G}_{m, n}$ works, but as just seen some of the polynomials in $\mathscr{G}_{m, n}$ may vanish modulo $U$.

To begin with, notice that if $m+n \leqslant 3$ then $\Gamma_{m, n} \cap T_{2}(B)=0=\Gamma_{m, n} \cap T_{2}(A)$, so $\Gamma_{m, n}(B)=\Gamma_{m, n}(A)$. Moreover, any $A$-valued substitution $\varphi_{A}$ gives rise to a $B$ valued substitution $\varphi_{B}=\Theta \varphi_{A}$, and all substitutions $\varphi: F\langle X\rangle \rightarrow B$ are obtained this way. This fact will significantly simplify the computations.

It is easy to check that 
Lemma 12. Let $m \geqslant 4$. Then $\mathscr{G}_{m, 0}$ is linearly independent modulo $T_{2}(B)$. In particular, $\Gamma_{m, 0} \cap U=\Gamma_{m, 0} \cap T_{2}(B)$.

Proof. The set $\mathscr{G}_{m, 0}$ spans $\Gamma_{m, 0}(B)$ and none of its elements is in $U$, since the new identities in $\mathscr{C}$ must involve at least one $z$. Moreover, it is linearly independent modulo $T_{2}(B)$, by the same reasoning in the proof of Lemma 5 , and the same substitutions provide the needed $B$-valued substitutions simply by composing them with $\Theta$, that is

$$
\begin{array}{ll}
y & Y_{(1)} \\
\mathbf{s}_{12}^{\prime} & \mathbf{u}_{2}^{\prime}
\end{array} \text { and } \begin{array}{llll}
y & Y_{(1)} & \bar{y} & Y_{(2)} \\
\mathbf{s}_{12}^{\prime} & \mathbf{u}_{1}^{\prime} & \mathbf{s}_{23}^{\prime} & \mathbf{u}_{3}^{\prime}
\end{array}
$$

Lemma 13. If $m \geqslant 3$ the polynomials $s(z, x), \bar{s}(z, x)$ and $c(z)$ form a basis of $\Gamma_{m, 1}(B)$. In particular, $\Gamma_{m, 1} \cap U=\Gamma_{m, 1} \cap T_{2}(B)$.

Proof. $\mathscr{G}_{m, 1}$ spans $\Gamma_{m, 1}(A)$, hence it spans also $\Gamma_{m, 1}(B)$; by the way the polynomials $d\left(x_{1}, Y_{(1)}, x_{2}\right)$ have $\mathbb{Z}_{2}$-degree 1 , hence just the polynomials of type $s, \bar{s}, c$ are actually involved in spanning $\Gamma_{m, 1}(B)$. To prove that they are linearly independent modulo $T_{2}(B)$ it is enough to compose with $\Theta$ the substitutions listed in the proof of Lemma 6.

The proof of the next result follows the same line, and we omit it.

Lemma 14. Let $m \geqslant 2$. Then $\mathscr{G}_{m, 2}$ is linearly independent modulo $T_{2}(B)$. In particular, $\Gamma_{m, 2} \cap U=\Gamma_{m, 2} \cap T_{2}(B)$.

More caution is needed if $n \geqslant 3$ :

Lemma 15. Let $m \geqslant 1, n \geqslant 3$.

- If $n \equiv 0(\bmod 2)$ then $\mathscr{G}_{m, n}$ is linearly independent modulo $T_{2}(B)$

- if $n \equiv 1(\bmod 2)$ then $\Gamma_{m, n}$ is spanned modulo $U$ by all the polynomials $s(x):=w\left[x, Y_{(1)}\right]$ and $\bar{s}(x):=\left[x, Y_{(1)}\right] w$; they are linearly independent modulo $T_{2}(B)$.

In particular, $\Gamma_{m, n} \cap U=\Gamma_{m, n} \cap T_{2}(B)$.

Proof. Since at least three $z$ 's are involved, the polynomials $d\left(x_{1}, Y_{(1)}, x_{2}\right)=\left[x_{1}, Y_{(1)}\right] w\left[x_{2}, Y_{(2)}\right]$, $s(z, x)=z w\left[x, Y_{(1)}\right]$ and $\bar{s}(z, x)=\left[x, Y_{(1)}\right] w z$ have $l(w) \geqslant 1$, and their classes span $\Gamma_{m, n}$ both $\bmod U$ and $\bmod T_{2}(B)$ because they span $\Gamma_{m, n}$ modulo $T_{2}(A)$. If $n \equiv 0(\bmod 2)$ then the usual steps are available. So let us assume $n \equiv 1(\bmod 2)$, hence the polynomials of type $d$ are in $U$ and just the polynomials of type $s(z, x)$ and $\bar{s}(z, x)$ suffices to span $\Gamma_{m, n}$ modulo $U$. By the way, they are not linearly independent, not even modulo $U$ : for fixed $s(z, x)=z w\left[x, Y_{(1)}\right]$ let $z_{0}$ be the smallest letter among $z$ and those occurring in $w$. If $z \neq z_{0}$ we may write

$z w\left[x, Y_{(1)}\right]=z z_{0} w^{\prime}\left[x, Y_{(1)}\right]=z_{0} z w^{\prime}\left[x, Y_{(1)}\right]+\left[z, z_{0}\right] w\left[x, Y_{(1)}\right] \equiv z_{0} z w^{\prime}\left[x, Y_{(1)}\right] \bmod U$

since the second summand has odd $\mathbb{Z}_{2}$-degree and so it is a consequence of $\mathscr{C}$. Hence we just need the polynomials of type $s(x):=w\left[x, Y_{(1)}\right]$ and, similarly, of type $\bar{s}(x):=\left[x, Y_{(1)}\right] w$ to span $\Gamma_{m, n}$ modulo $U$. Now we are going to test their linear independence $\bmod T_{2}(B)$. This is actually easy: just set

$$
\varphi:=\begin{array}{ccc}
w & x & Y_{(1)} \\
\mathbf{v}^{\prime} & \mathbf{x}_{23}^{\prime} & \mathbf{u}_{3}^{\prime}
\end{array}
$$


and notice that $\varphi(s(x)) \neq \mathbf{0}$ while all other polynomials $s\left(x^{\prime}\right), \bar{s}\left(x^{\prime}\right)$ are $\mathbf{0}$-valued. Next the polynomials $\bar{s}(x)$ are dealt with similarly.

Notice also that the case $m=1, n \geqslant 3$ poses no difficulties: simply there are no polynomials $d\left(x_{1}, Y_{(1)}, x_{2}\right)$ in $\mathscr{G}_{1, n}$, the rest is the same.

The last case, when just letters from $Z$ occur, deserves more care:

Lemma 16. Let $n \geqslant 4$. If $n \equiv 0(\bmod 2)$ then $\mathscr{G}_{0, n}$ is linearly independent modulo $U$. If $n \equiv 1(\bmod 2)$ then the polynomial $w_{0}:=z_{1} \ldots z_{n}$ and the polynomials of type $\left[z, z_{1}\right] w, w\left[z_{n}, z\right]$ form a set spanning $\Gamma_{0, n}$ modulo $U$ and linearly independent modulo $T_{2}(B)$.

In particular in both cases $\Gamma_{0, n} \cap U=\Gamma_{0, n} \cap T_{2}(B)$.

Proof. If $n$ is even the statement follows easily, so assume $n$ is odd.

$\Gamma_{0, n}$ is spanned by the polynomials in $\mathscr{G}_{0, n}$ modulo $T_{2}(A)$, hence the same set spans $\Gamma_{0, n}$ modulo $U$ as well. We are going to prove that each $f \in \mathscr{G}_{0, n}$ is a linear combination of $w_{0},\left[z, z_{1}\right] w, w\left[z_{n}, z\right]$ modulo $U$.

Let us denote $V=\operatorname{span}_{F}\left\langle w_{0},\left[z, z_{1}\right] w, w\left[z_{n}, z\right] \mid z_{1}<z<z_{n}\right\rangle$, let $f=z w \bar{z}$ be a fixed polynomial in $\mathscr{G}_{0, n}$ and let $\operatorname{pos}\left(z_{i}\right)$ denote the position of the letter $z_{i}$ in $f$. Since $w$ is standard, $\operatorname{pos}\left(z_{1}\right) \in\{1,2, n\}$. Consider the possible values of $\operatorname{pos}\left(z_{1}\right)$ :

- $\operatorname{pos}\left(z_{1}\right)=1$. Then $\operatorname{pos}\left(z_{n}\right) \in\{n-1, n\}$.

- If $\operatorname{pos}\left(z_{n}\right)=n$ then $f=z_{1} w z_{n}$ and $f=w_{0}$;

- if $\operatorname{pos}\left(z_{n}\right)=n-1$ then $f=z_{1} w^{\prime} z_{n} \bar{z}$ and we get

$$
f=z_{1} w^{\prime} \bar{z} z_{n}+z_{1} w^{\prime}\left[z_{n}, \bar{z}\right] \equiv w_{0}+z_{1} w^{\prime}\left[z_{n}, \bar{z}\right] \bmod U,
$$

with both the summands in $V$.

- $\operatorname{pos}\left(z_{1}\right)=2$. Now $\operatorname{pos}\left(z_{n}\right) \in\{1, n-1, n\}$ :

- if $\operatorname{pos}\left(z_{n}\right)=1$ then $f=z_{n} z_{1} w_{1} \bar{z}$ and

$$
f=z_{1} z_{n} w_{1} \bar{z}+\left[z_{n}, z_{1}\right] w_{1} \bar{z} \equiv z_{1} w_{1} z_{n} \bar{z}+\left[z_{n}, z_{1}\right] w_{2} \quad \bmod U
$$

hence $f \in(V+U) / U$. In fact in the first summand $\operatorname{pos}\left(z_{1}\right)=1$, so it is in $(V+U) / U$ by the previous case, while in the second summand if $\bar{z} \neq z_{n-1}$ the straightening is possible because of the identity $\left[x_{1}, x_{2}\right] w\left[x_{3}, x_{4}\right] \in U($ recall $\partial(f)=1)$;

- if $\operatorname{pos}\left(z_{n}\right)=n-1$ it is $f=z z_{1} w_{1} z_{n} \bar{z}$. Then flipping $z$ and $z_{1}$ we get

$$
f=z_{1} z w_{1} z_{n} \bar{z}+\left[z, z_{1}\right] w_{1} z_{n} \bar{z} \equiv z_{1} w_{2} z_{n} \bar{z}+\left[z, z_{1}\right] w_{3} \quad(\bmod U)
$$

which is in $(V+U) / V$ for the same reasons as before;

- if $\operatorname{pos}\left(z_{n}\right)=n$ it is $f=z z_{1} w_{1} z_{n}$ and is enough to notice $f=z_{1} z w_{1} z_{n}+$ $\left[z, z_{1}\right] w_{1} z_{n}$.

- $\operatorname{pos}\left(z_{1}\right)=n$. Then $\operatorname{pos}\left(z_{n}\right) \in\{1, n-1\}$. The case $\operatorname{pos}\left(z_{n}\right)=n-1$ is easy: $f=z w z_{n} z_{1}$ so exchange $z_{1}$ and $z_{n}$ by the basic commutation rule, as before. Instead the case $\operatorname{pos}\left(z_{n}\right)=1$, that is when $f=z_{n} w z_{1}$, is more subtle. Write $w=z_{2} w_{2} z_{n-1}$ and let us work modulo $(V+U) / U$. We have

$$
\begin{aligned}
f & =z_{2} z_{n} w_{2} z_{n-1} z_{1}+\left[z_{n}, z_{2}\right] w_{2} z_{n-1} z_{1} \equiv z_{2} w_{2} z_{n-1} z_{n} z_{1}+\left[z_{n}, z_{2}\right] z_{1} w_{2} z_{n-1} \\
& =z_{2} w_{2} z_{n-1} z_{1} z_{n}+z_{2} w_{2} z_{n-1}\left[z_{n}, z_{1}\right]+z_{1}\left[z_{n}, z_{2}\right] w_{2} z_{n-1}+\left[z_{n}, z_{2}, z_{1}\right] w_{2} z_{n-1} \\
& \equiv\left[z_{n}, z_{2}, z_{1}\right] w_{2} z_{n-1}
\end{aligned}
$$


because the first and the second summand are in $V+U$ and the third in $U$. Now, by the Jacobi identity $[a, b, c]+[b, c, a]+[c, a, b]=0$, it follows

$$
\begin{aligned}
f & =\left[z_{1}, z_{2}, z_{n}\right] w_{2} z_{n-1}+\left[z_{n}, z_{1}, z_{2}\right] w_{2} z_{n-1} \\
& \equiv\left[z_{1}, z_{2}\right] z_{n} w_{2} z_{n-1}+\left[z_{n}, z_{1}\right] z_{2} w_{2} z_{n-1} \equiv 0
\end{aligned}
$$

Now it is easy to show that the polynomials $w_{0},\left[z, z_{1}\right] w$ e $w\left[z, z_{n}\right]$ are linearly independent modulo $T_{2}(B)$ : just $w_{0}$ survives under the substitution sending all letters to $\mathbf{v}^{\prime}$, so it is linearly independent with the other polynomials; then the substitution sending $z \rightarrow \mathbf{t}_{12}^{\prime}$ and the other letters in $\mathbf{v}^{\prime}$ saves just $\left[z, z_{1}\right] w$, and finally the substitution sending $z \rightarrow \mathbf{t}_{23}^{\prime}$ and all other letters in $\mathbf{v}^{\prime}$ saves just $w\left[z, z_{n}\right]$.

So the task is done:

Corollary 17. $U=T_{2}(B)$. Moreover, $T_{2}(B)=T_{2}(A)+(\mathscr{C})^{T_{2}}$.

\section{Proper multilinear spaces for $A$ And $B$}

The vector space $\Gamma_{m, n}$ has a natural $S_{m} \times S_{n}$-left module structure, with $S_{m}$ renaming the indeterminates $y_{1}, \ldots, y_{m}$ and $S_{n}$ renaming $z_{1}, \ldots, z_{n}$. For any $T_{2^{-}}$ ideal $T, \Gamma_{m, n} \cap T$ is a submodule of $\Gamma_{m, n}$, so the factor space is canonically turned into an $S_{m} \times S_{n}$-module. Here we are interested in describing the module structure of the factor modules $\Gamma_{m, n}(R)=\Gamma_{m, n} /\left(\Gamma_{m, n} \cap T_{2}(R)\right)$ for $R=A, B$ and their dimensions (the proper codimensions $\gamma_{m, n}(A)$ and $\left.\gamma_{m, n}(B)\right)$. We recall that the distinct isomorphism classes of irreducible $S_{m} \times S_{n}$-modules are in a one-to-one correspondence with the pair of partitions $(\lambda, \mu), \lambda \vdash m$ and $\mu \vdash n$; moreover, if $M_{\alpha}$ denotes a representative for the isomorphism class corresponding to the partition $\alpha \vdash a$, then any representative for the $S_{m} \times S_{n}$-irreducible modules corresponding to the pair $(\lambda, \mu)$ is isomorphic to the tensor product $M_{\lambda} \otimes M_{\mu}$. We shall abuse the notation and write $\lambda \otimes \mu$ to denote $M_{\lambda} \otimes M_{\mu}$, in order to keep the notation as simple as possible. For the same reason, if $\alpha \vdash a$ and $\beta \vdash b$, the induced module $\left(M_{\alpha} \otimes M_{\beta}\right)^{S_{a+b}}$ will be simply denoted by $(\alpha \otimes \beta)^{S_{a+b}}$.

In order to describe the structure module of $\Gamma_{m, n}(A)$ and $\Gamma_{m, n}(B)$ we may clearly assume $m+n \geqslant 4$, since if $m+n \leqslant 3$ then $\Gamma_{m, n}(A)=\Gamma_{m, n}(B)=\Gamma_{m, n}$.

Proposition 18. For all $m \geqslant 4$ it holds

$$
\Gamma_{m, 0}(A)=\Gamma_{m, 0}(B) \cong\left((m-1,1) \oplus \bigoplus_{l=2}^{m-2}((l-1,1) \otimes(m-l-1,1))^{S_{m}}\right) \otimes \varnothing
$$

In particular, $\gamma_{m, 0}(A)=\gamma_{m, 0}(B)=\left(2^{m-2}(m-4)+3\right)(m-1)$.

Proof. We already know that $\Gamma_{m, 0}(A)=\Gamma_{m, 0}(B)$; since no $z$ occurs, this space is the tensor product of the proper part of the (ordinary) algebra $U T_{3}(F)$ with the trivial module $\varnothing$. So it is a known result (see [16]). Then

$$
\gamma_{m, 0}(A)=(m-1)+\sum_{l=2}^{m-2}\left(\begin{array}{c}
m \\
l
\end{array}\right)(l-1)(m-l-1),
$$

and the result follows. 
Proposition 19. For $n=1$ it holds

$$
\begin{aligned}
& \Gamma_{3,1}(A) \cong 2\left(\left(\left(1^{2}\right) \otimes(1)\right)^{S_{3}} \otimes(1)\right) \oplus 2((2,1) \otimes(1)) \oplus((3) \otimes(1)) \\
& \Gamma_{3,1}(B) \cong 2((2,1) \otimes(1)) \oplus((3) \otimes(1))
\end{aligned}
$$

and if $m>3$ then

$$
\begin{aligned}
\Gamma_{m, 1}(A) \cong & \left(\bigoplus_{l=2}^{m-2}((l-1,1) \otimes(m-l-1,1))^{S_{m}} \otimes(1)\right) \oplus \\
& 2\left(\bigoplus_{l=2}^{m-1}((l-1,1) \otimes(m-l))^{S_{m}} \otimes(1)\right) \oplus 2((m-1,1) \otimes(1)) \oplus((m) \otimes(1)) \\
\Gamma_{m, 1}(B) \cong 2 & ((m-1,1) \otimes(1)) \oplus((m) \otimes(1))
\end{aligned}
$$

In particular, for all $m \geqslant 3$ it is

$$
\gamma_{m, 1}(A)=2^{m-2}\left(m^{2}-m-4\right)+2 m+1, \quad \gamma_{m, 1}(B)=2 m-1 .
$$

Proof. Assume $m>3$, since the proof when $m=3$ uses just partial arguments of the general case.

Fix any $2 \leqslant l \leqslant m-2$, set $d_{l}=\left[y_{2}, y_{1}, \ldots, y_{l}\right] z\left[y_{l+2}, y_{l+1}, \ldots, y_{m}\right]$ and denote $H_{l}=S_{l} \times \operatorname{Sym}(\{l+1, \ldots, m\}) \leqslant S_{m}$. The polynomial $d_{l}$ generates a $F\left(H_{l} \times S_{1}\right)$ module isomorphic to $((l-1,1) \otimes(m-l-1,1)) \otimes(1)$, since triple commutators are in $T_{2}(A)$. The $S_{m} \times S_{1}$-module generated by $d_{l}$ contains all the basis elements of $\Gamma_{m, 1}(A)$ which are product of a commutator of length $l, z$ and a commutator of length $m-l$. Their number is $\left(\begin{array}{c}m \\ l\end{array}\right)(l-1)(m-l-1)=\operatorname{dim}(((l-1,1) \otimes(m-l-$ $\left.1,1))^{S_{m}} \otimes(1)\right)$, so it is isomorphic to the full induced module. Varying $l$ we get the first summand of the statement.

Now, for any fixed $2 \leqslant l \leqslant m-1$ define $u_{l}=\left[y_{2}, y_{1}, y_{3}, \ldots, y_{l}\right]\left[z, y_{l+1}, \ldots, y_{m}\right]$ and set $K_{l}=S_{l} \times \operatorname{Sym}(\{l+1, \ldots, m\}) \leqslant S_{m}$. Then $u_{l}$ generates an $F\left(K_{l} \times S_{1}\right)$ module isomorphic to $((l-1,1) \otimes(m-l)) \otimes(1)$ in $\Gamma_{m, 1}(A)$ (we remark that it is not a submodule of $\left.\Gamma_{m, 1}\right)$ and as before, looking at the $S_{m} \times S_{1}$ action, we get a module isomorphic to $((l-1,1) \otimes(m-l))^{S_{m}} \otimes(1)$. It has an isomorphic copy, namely the one obtained by $\left[z, y_{l+1}, \ldots, y_{m}\right]\left[y_{2}, y_{1}, y_{3}, \ldots, y_{l}\right]$. Varying $l$ we get direct summands of $\Gamma_{m, 1}(A)$, and second summand in the statement then follows.

Let $W$ be the direct sum of the submodules so far obtained. In the factor module $\Gamma_{m, 1}(A) / W$ we have the submodules generated by $\left[y_{2}, y_{1}, \ldots, y_{m}\right] z+W$, $z\left[y_{2}, y_{1}, \ldots, y_{m}\right]+W$ and $\left[z, y_{1}, \ldots, y_{m}\right]+W$, which are clearly disjoint and isomorphic to $(m-1,1) \otimes(1)$ (the first pair) and $(m) \otimes(1)$ (the latter). By complete reducibility and dimensional arguments, we get finally the stated decomposition of $\Gamma_{m, 1}(A)$.

Passing to $\Gamma_{m, 1}(B)$, just notice that all $d_{l}$ 's and $u_{l}$ 's (together with their specular polynomials) are in $T_{2}(B)$, so the related modules lay inside $\Gamma_{m, 1} \cap T_{2}(B)$. By dimensional arguments we get the stated decomposition of $\Gamma_{m, 1}(B)$. 
In particular, if $m \geqslant 4$ then

$$
\begin{aligned}
\gamma_{m, 1}(A) & =\sum_{l=2}^{m-2}\left(\begin{array}{c}
m \\
l
\end{array}\right)(l-1)(m-l-1)+2 \sum_{l=2}^{m-1}\left(\begin{array}{c}
m \\
l
\end{array}\right)(l-1)+2(m-1)+1 \\
& =2^{m-2}\left(m^{2}-m-4\right)+2 m+1 \\
\gamma_{m, 1}(B) & =2(m-1)+1=2 m-1
\end{aligned}
$$

and the particular cases $\gamma_{3,1}(A)$ and $\gamma_{3,1}(B), 11$ and 5 respectively, also follow from the general formula, as one may check.

The other cases admit very similar arguments. Essentially, the polynomials of type $d, s$ and $\bar{s}$ lead to $F\left(S_{m} \times S_{n}\right)$-submodules of $\Gamma_{m, n}(A)$ (or $B$ ), starting from subgroups $H \times K$ actions (for suitable $H \leqslant S_{m}$ and $K \leqslant S_{n}$ ), then looking at the $S_{m} \times S_{n}$ actions. Factoring out the sum $W$ of such submodules of $\Gamma_{m, n}(A)$ the factor module $\Gamma_{m, n}(A) / W$ is a direct sum of modules generated by the classes $c(x)+W$. Then complete reducibility and dimensional comparisons provide the whole $S_{n} \times S_{m}$-structure of $\Gamma_{m, n}(A)$ and $\Gamma_{m, n}(B)$. Notice that if $n \equiv 0(\bmod 2)$ then $\Gamma_{m, n}(A) \cong \Gamma_{m, n}(B)$, while if $n \equiv 1(\bmod 2)$ the nontrivial module $W \cap T_{2}(B)$ causes a drastic reduction of irreducible summands in $\Gamma_{m, n}(B)$. Once the structure is known, the proper codimension sequence follows quite easily.

Since this will be the common path, in the proofs of the following results we shall just list the starting polynomials, with their multiplicities. We also have to keep an eye on the particular cases, for small values of $m$ or $n$.

Proposition 20. If $m \geqslant 2$ and $n=2$ it is $\Gamma_{m, 2}(A)=\Gamma_{m, n}(B)$; more precisely

$$
\begin{aligned}
\Gamma_{2,2}(A) \cong & 3((2) \otimes(2)) \oplus 3\left((2) \otimes\left(1^{2}\right)\right) \oplus 3\left(\left(1^{2}\right) \otimes(2)\right) \oplus 3\left(\left(1^{2}\right) \otimes\left(1^{2}\right)\right) \\
\Gamma_{3,2}(A) \cong & 4((3) \otimes(2)) \oplus 4\left((3) \otimes\left(1^{2}\right)\right) \oplus 6((2,1) \otimes(2)) \oplus 6\left((2,1) \otimes\left(1^{2}\right)\right) \\
& \oplus 2\left(\left(1^{3}\right) \otimes(2)\right) \oplus 2\left(\left(1^{3}\right) \otimes\left(1^{2}\right)\right)
\end{aligned}
$$

and, if $m>3$,

$$
\begin{aligned}
\Gamma_{m, 2}(A) \cong & \left(\bigoplus_{l=2}^{m-2}((l-1,1) \otimes(m-l-1,1))^{S_{m}} \otimes(2)\right) \oplus \\
& 2\left(\bigoplus_{l=2}^{m-1}((l-1,1) \otimes(m-l))^{S_{m}} \otimes\left((2) \oplus\left(1^{2}\right)\right)\right) \oplus \\
& \left(\bigoplus_{l=1}^{m-1}((l) \otimes(m-l))^{S_{m}} \otimes\left((2) \oplus\left(1^{2}\right)\right)\right) \oplus \\
& 2\left((m-1,1) \otimes\left((2) \oplus\left(1^{2}\right)\right)\right) \oplus 2\left((m) \otimes\left((2) \oplus\left(1^{2}\right)\right)\right)
\end{aligned}
$$

In particular, for all $m \geqslant 2, \gamma_{m, 2}(A)=\gamma_{m, 2}(B)=2^{m-2}(m+4)(m-1)+2(m+1)$. 
Proof. The list of starting polynomials, modules and multiplicities is summarized in the following table. Clearly, the multiplicities depend on the position of the $z$ 's.

\begin{tabular}{|l|l|c|l|}
\hline condition & polynomial & copies & $\left(S_{m} \times S_{2}\right)$ - module \\
\hline \hline $\begin{array}{l}m \geqslant 4 \\
2 \leqslant l \leqslant m-2\end{array}$ & {$\left[y_{2}, y_{1}, \ldots, y_{l}\right] z_{1} z_{2}\left[y_{l+2}, y_{l+1}, \ldots, y_{m}\right]$} & 1 & $((l-1,1) \otimes(m-l-1,1))^{S_{m}} \otimes(2)$ \\
\hline $\begin{array}{l}m \geqslant 3 \\
2 \leqslant l \leqslant m-1\end{array}$ & {$\left[y_{2}, y_{1}, \ldots, y_{l}\right] z_{2}\left[z_{1}, y_{l+1}, \ldots, y_{m}\right]$} & 2 & $((l-1,1) \otimes(m-l))^{S_{m}} \otimes((1) \otimes(1))^{S_{2}}$ \\
\hline $1 \leqslant l \leqslant m-1$ & {$\left[z_{1}, y_{1}, \ldots, y_{l}\right]\left[z_{2}, y_{l+1}, \ldots, y_{m}\right]$} & 1 & $((l) \otimes(m))^{S_{m}} \otimes((1) \otimes(1))^{S_{2}}$ \\
\hline & {$\left[y_{2}, y_{1}, \ldots, y_{m}\right] z_{1} z_{2}$} & 2 & $(m-1,1) \otimes((1) \otimes(1))^{S_{2}}$ \\
\hline & {$\left[z_{1}, y_{1}, \ldots, y_{m}\right] z_{2}$} & 2 & $(m) \otimes((1) \otimes(1))^{S_{2}}$ \\
\hline
\end{tabular}

Proposition 21. For $m \geqslant 1$ and $n \geqslant 3$ it is

$$
\begin{aligned}
& \Gamma_{1, n}(A) \cong 2\left((1) \otimes\left(F S_{2} \otimes(n-2)\right)^{S_{n}}\right) \\
& \Gamma_{2, n}(A) \cong\left(F S_{2} \otimes\left(F S_{2} \otimes(n-2)\right)^{S_{n}}\right) \oplus 2\left(\left(1^{2}\right) \otimes((1) \otimes(n-1))^{S_{n}}\right) \\
& \oplus 2\left((2) \otimes\left(F S_{2} \otimes(n-2)\right)^{S_{n}}\right) \\
& \Gamma_{3, n}(A) \cong 2\left(\left(\left(1^{2}\right) \otimes(1)\right)^{S_{3}} \otimes((n-1) \otimes(1))^{S_{n}}\right) \oplus 2\left(((2) \otimes(1))^{S_{3}} \otimes\left(F S_{2} \otimes(n-2)\right)^{S_{n}}\right) \\
& \oplus 2\left((2,1) \otimes((1) \otimes(n-1))^{S_{n}}\right) \oplus 2\left((3) \otimes\left(F S_{2} \otimes(n-2)\right)^{S_{n}}\right)
\end{aligned}
$$

and, if $m>3$,

$$
\begin{aligned}
\Gamma_{m, n}(A) & \cong\left(\sum_{\substack{1 \leqslant h \leqslant m-3 \\
h+k=m-2}}((h, 1) \otimes(k, 1))^{S_{m}} \otimes(n)\right) \oplus 2\left(\sum_{\substack{1 \leqslant h \leqslant m-2 \\
h+k=m-1}}((h, 1) \otimes(k))^{S_{m}} \otimes((1) \otimes(n-1))^{S_{n}}\right) \\
& \oplus\left(\sum_{h=1}^{m-1}((h) \otimes(m-h))^{S_{m}} \otimes\left(F S_{2} \otimes(n-2)\right)^{S_{n}}\right) \\
& \oplus 2\left((m-1,1) \otimes((1) \otimes(n-1))^{S_{n}}\right) \oplus 2\left((m) \otimes\left(F S_{2} \otimes(n-2)\right)^{S_{n}}\right) .
\end{aligned}
$$

In particular, for all $m \geqslant 1$ and $n \geqslant 3$, it is

$$
\gamma_{m, n}(A)=2^{m-2}\left(m^{2}+4 m n+4 n^{2}-5 m-12 n+4\right)+2(m+n-1) .
$$


Proof. Here the list is

\begin{tabular}{|l|l|c|l|}
\hline condition & polynomials & copies & $\left(S_{m} \times S_{n}\right)$ - modules \\
\hline \hline $\begin{array}{l}m \geqslant 4 \\
2 \leqslant l \leqslant m-2\end{array}$ & {$\left[y_{2}, y_{1}, \ldots, y_{l}\right] z_{1} \ldots z_{n}\left[y_{l+2}, y_{l+1}, \ldots, y_{m}\right]$} & 1 & $((l-1,1) \otimes(m-l-1,1))^{S_{m}} \otimes(n)$ \\
\hline $\begin{array}{l}m \geqslant 3 \\
2 \leqslant l \leqslant m-1\end{array}$ & {$\left[y_{2}, y_{1}, \ldots, y_{l}\right] z_{2} \ldots z_{n}\left[z_{1}, y_{l+1}, \ldots, y_{m}\right]$} & 2 & $((l-1,1) \otimes(m-l))^{S_{m}} \otimes((n-1) \otimes(1))^{S_{n}}$ \\
\hline $\begin{array}{l}m \geqslant 2 \\
1 \leqslant l \leqslant m-1\end{array}$ & {$\left[z_{1}, y_{1}, \ldots, y_{l}\right] z_{3} \ldots z_{n}\left[z_{2}, y_{l+1}, \ldots, y_{m}\right]$} & 1 & $((l) \otimes(m))^{S_{m}} \otimes\left(F S_{2} \otimes(n-2)\right)^{S_{n}}$ \\
\hline$m \geqslant 2$ & {$\left[y_{2}, y_{1}, \ldots, y_{m}\right]\left(z_{2} \ldots z_{n}\right) z_{1}$} & 2 & $(m-1,1) \otimes((1) \otimes(n-1))^{S_{n}}$ \\
\hline & {$\left[z_{1}, y_{1}, \ldots, y_{m}\right]\left(z_{3} \ldots z_{n}\right) z_{2}$} & 2 & $(m) \otimes\left(F S_{2} \otimes(n-2)\right)^{S_{n}}$ \\
\hline
\end{tabular}

Proposition 22. Let $n \geqslant 3$ and $m \geqslant 1$.

If $n \equiv 0(\bmod 2)$ then $\Gamma_{m, n}(B)=\Gamma_{m, n}(A)$.

If $n \equiv 1(\bmod 2)$ then:

$$
\Gamma_{1, n}(B) \cong 2(((1) \otimes(n)) \oplus((1) \otimes(n-1,1)))
$$

and, for $m>1$,

$$
\Gamma_{m, n}(B) \cong 2(((m) \otimes(n)) \oplus((m) \otimes(n-1,1)) \oplus((m-1,1) \otimes(n)))
$$

In particular, if $n$ is odd, then $\gamma_{m, n}(B)=2(m+n-1)$.

Proof. If $n \equiv 1(\bmod 2)$ the list is:

\begin{tabular}{|l|l|c|l|}
\hline conditions & polynomial & copies & $\left(S_{m} \times S_{n}\right)-$ modules \\
\hline \hline$m \geqslant 2$ & {$\left[y_{2}, y_{1}, \ldots, y_{m}\right] z_{1} \ldots z_{n}$} & 2 & $(m-1,1) \otimes(n)$ \\
\hline & {$\left[z_{1}, y_{1}, \ldots, y_{m}\right] z_{2} \ldots z_{3}$} & 2 & $(m) \otimes((1) \otimes(n-1))$ \\
\hline
\end{tabular}

Finally,

Proposition 23. For any $n \geqslant 4$ it is

$$
\Gamma_{0, n}(A) \cong \varnothing \otimes\left((n) \oplus 2(n-1,1) \oplus(n-2,2) \oplus\left(n-2,1^{2}\right)\right) .
$$

If $n \equiv 0(\bmod 2)$ then $\Gamma_{0, n}(B)=\Gamma_{0, n}(A)$.

If $n \equiv 1(\bmod 2)$ then $\Gamma_{0, n}(B) \cong \varnothing \otimes((n) \oplus 2(n-1,1))$.

In particular, $\gamma_{0, n}(A)=n(n-1)$ and, if $n$ is odd, then $\gamma_{0, n}(B)=2 n-1$.

Proof. In $\Gamma_{0, n}(A)$ just the polynomial $z_{n-1} w z_{n}$ suffices to generate an $S_{n-2} \times$ $\operatorname{Sym}(\{n-1, n\})$-submodule isomorphic to $(n-2) \otimes F S_{2}$, and $F S_{2} \cong S_{2}(2) \otimes\left(1^{2}\right)$. Then, looking at the $S_{n}$-module, the statement follows both for $\Gamma_{0, n}(A)$ and $\Gamma_{0, n}(B)$ if $n \equiv 0(\bmod 2)$.

If instead we assume $n \equiv 1(\bmod 2)$, then the polynomials $\left[z_{2}, z_{1}\right] w$ and $w\left[z_{2}, z_{1}\right]$ generate two copies of the $S_{2} \times S_{n-2}$-module isomorphic to $\left(1^{2}\right) \otimes(n-2)$; looking 
to the $S_{n}$-modules, their direct sum is $W \cong_{S_{n}} 2(n-1,1)$. The factor module can be generated by $z_{1} \ldots z_{n}+W$ and is isomorphic to $(n)$. The proper codimensions follow easily.

\section{Codimension sequences for $A$ And $B$}

The proper multilinear spaces $\Gamma_{m, n}(A)$ and $\Gamma_{m, n}(B)$ provide full knowledge of the relatively free algebras of the involved $T_{2}$-ideal, not just in principle. Hence the comparisons between their structures and their dimensions are already very significant. In particular we may already see the drastic structure simplification of $\Gamma_{m, n}(B)$ compared to $\Gamma_{m, n}(A)$ when $n$ is odd, causing a radical slowdown in the codimension growth of $B$ with respect to the codimension growth of $A$.

By the way, there are more standard invariants to be compared, mainly the codimension sequences $c_{m, n}(A)$ and $c_{m, n}(B)$, the (more important) $\mathbb{Z}_{2}$-codimension sequences $c_{n}^{\mathbb{Z}_{2}}(A)$ and $c_{n}^{\mathbb{Z}_{2}}(B)$, and the cocharacter sequences $\chi_{m, n}(A)$ and $\chi_{m, n}(B)$ (the hyperoctahedral-related cocharacter sequences $\chi_{n}^{\mathbb{Z}_{2}}(A)$ and $\chi_{n}^{\mathbb{Z}_{2}}(B)$ are essentially the same as the mentioned cocharacter sequences). This, of course, does not mean those invariants are easily recovered by the proper ones. In this section, we shall explicitly compute the $c_{m, n}$ and the $c_{n}^{\mathbb{Z}_{2}}$ codimensions for $A$ and $B$.

Recall that for any superalgebra $R$ the $(m, n)$-th partial codimension is $c_{m, n}(R)=$ $\operatorname{dim} P_{m, n}(R)$, and there is a precise relation ([17], Prop. 1, (3)) between $c_{m, n}(R)$ and $\gamma_{m, n}(R)$, holding for all $m, n \in \mathbb{N}$ :

$$
c_{m, n}(R)=\sum_{h=0}^{m}\left(\begin{array}{c}
m \\
h
\end{array}\right) \gamma_{h, n}(R) .
$$

Here, for all $m \in \mathbb{N}$ and all even $n \in \mathbb{N}$ clearly one has $c_{m, n}(A)=c_{m, n}(B)$, while there are significative differences when $n$ is odd.

For convenience of the reader, we summarize the proper codimension sequences obtained in the last section. We may organize the data in a matrix whose $(i, j)$-entry is $\gamma_{i, j}$

- 1 is the entry in $(0,0)$ and $(0,1)$, holding for both $A$ and $B$. It cannot be recovered from the general formulas ruling the sequences along the first two columns and the first row, and actually it is a singular value to be taken into account in deriving the $c_{m, n}$ 's;

- the remaining entries on the first row, that are $\gamma_{0, n}$ for $n \geqslant 2$, are $\gamma_{0, n}(A)=$ $n(n-1)$ and for odd $n$ it is $\gamma_{0, n}(B)=2 n-1$.

- The entries $\gamma_{h, 0}$ for $h \geqslant 1$ are the same for $A$ and $B$; precisely $\gamma_{h, 0}=$ $\left(2^{h-2}(h-4)+3\right)(h-1)$;

- the entries $\gamma_{h, 1}$, for $h \geqslant 1$, differ for $A$ and $B$. Precisely $\gamma_{h, 1}(A)=2^{h-2}\left(h^{2}-\right.$ $h-4)+2 h+1, \gamma_{h, 1}(B)=2 h-1$;

- all the other entries, that is for $h \geqslant 1$ and $n \geqslant 2$, follow a common rule:

$\gamma_{h, n}(A)=2^{h-2}\left(h^{2}+4 h n+4 n^{2}-5 h-12 n+4\right)+2(h+n-1), \gamma_{h, n}(B)=2(h+n-1)(n$ odd $)$

Now we can compute the $c_{m, n}$-values:

Theorem 24. The sequence $\left(c_{m, n}(A)\right)_{m, n \in \mathbb{N}}$ is the following: $c_{0,0}(A)=c_{0,1}(A)=1$ and, for all $m \in \mathbb{N}, n \geqslant 2$,

$$
c_{m, n}(A)=3^{m-2}\left((m+3 n)^{2}-7 m-27 n+9\right)+2^{m}(m+2 n-2)+1 .
$$


The sequence $\left(c_{m, n}(B)\right)_{m, n \in \mathbb{N}}$ is the following: $c_{0,0}(B)=c_{0,1}(B)=1$, and for all other $m, n \in \mathbb{N}, c_{m, n}(B)=c_{m, n}(A)$ if $n$ is even, while

$$
c_{m, 1}(B)=2^{m}(m-1)+2, \quad c_{m, n}(B)=2^{m}(m+2 n-2)+1(\text { for } n \geqslant 3)
$$

if $n$ is odd.

Proof. One separately computes the values $c_{m, 0}(A), c_{m, 1}(A)$ and $c_{m, n}(A)$ for $n \geqslant 2$, because the proper codimensions follow different rules, paying attention to the singular values $\gamma_{0, n}$, because they do not follow the general rule. So for instance we have

$$
c_{m, 0}(A)=\gamma_{0,0}(A)+\sum_{h=1}^{m}\left(\begin{array}{c}
m \\
h
\end{array}\right) \gamma_{h, 0}(A)=1+\sum_{h=1}^{m}\left(\begin{array}{c}
m \\
h
\end{array}\right)\left(2^{h-2}(h-4)+3\right)(h-1) .
$$

Elementary manipulations (splitting the sum into simpler sums, changing suitably the range of $h$, etc.) and the basic binomial expansion $(1+x)^{m}=\sum_{h=0}^{m}\left(\begin{array}{c}m \\ h\end{array}\right) x^{h}$, together with its derived expansions, provide the stated number.

All remaining cases for $A$ and $B$ are similar.

Remark 25. Notice that there is no apparent reason to get a unique formula for $c_{m, n}(A)$ (and indeed $c_{m, 1}(B)$ cannot be recovered from the more general $c_{m, n}(B)$ holding for odd $n \geqslant 3)$, but it turns out that $c_{m, 0}(A)$ and $c_{m, 1}(A)$ follow from the general rule $c_{m, n}(A)$ (so just $c_{0,0}(A)$ and $c_{0,1}(A)$ are exceptional).

Now we can obtain $c_{n}^{\mathbb{Z}_{2}}(A)=\operatorname{dim} P_{n}(A)$ : this will pose no difficulties but lengthy computations. Recall ([3], [5]) that $c_{n}^{\mathbb{Z}_{2}}(A)=\sum_{h=0}^{n}\left(\begin{array}{l}n \\ h\end{array}\right) \gamma_{h, n-h}(A)$.

Theorem 26. It is $c_{0}^{\mathbb{Z}_{2}}(A)=1, c_{1}^{\mathbb{Z}_{2}}(A)=2$ and, for $n \geqslant 2$,

$$
c_{n}^{\mathbb{Z}_{2}}(A)=4^{n-1}\left(n^{2}-5 n+4\right)+3^{n-1}(4 n-6)+2^{n} n+2 .
$$

More subtle is to compute $c_{n}^{\mathbb{Z}_{2}}(B)$ :

Theorem 27. It is $c_{0}^{\mathbb{Z}_{2}}(B)=1, c_{1}^{\mathbb{Z}_{2}}(B)=2$ and, for $n \geqslant 2$,

$$
c_{n}^{\mathbb{Z}_{2}}(B)=\frac{4^{n-1}}{2}\left(n^{2}-5 n+4\right)+3^{n-1}(4 n-6)+2^{n-1}+n+2 .
$$

Proof. We must compute

$$
c_{n}^{\mathbb{Z}_{2}}(B)=\sum_{h=0}^{n}\left(\begin{array}{l}
n \\
h
\end{array}\right) c_{h, n-h}(B) .
$$

While in the previous Theorem there was a unique expression for all $c_{h, n-h}(A)$, here we must consider separately the two last summands, that is

$$
c_{n}^{\mathbb{Z}_{2}}(B)=c_{n, 0}(B)+n c_{n-1,1}(B)+\sum_{h=0}^{n-2}\left(\begin{array}{l}
n \\
h
\end{array}\right) c_{h, n-h}(B) .
$$

We record

$$
c_{n, 0}(B)+n c_{n-1,1}(B)=3^{n-2}\left(n^{2}-7 n+9\right)+2^{n-1}\left(n^{2}+n-6\right)+2 n+3 .
$$

Then, we face the problem that the coefficients $c_{h, n-h}(B)$ depend on the parity of $n-h$. Let us define the parity map $\rho$ assigning to $k \in \mathbb{N}$ the remainder of $k: 2$. 
Hence $\rho(k) \neq 0$ if and only if $k$ is odd, and for $k \geqslant 2$ we can glue even and odd cases in $c_{h, k}(B)$ writing

$$
\begin{aligned}
c_{h, k}(B) & =\left(3^{h-2}\left((h+3 k)^{2}-7 h-27 k+9\right)+2^{h}(h+2 k-2)+1\right) \rho(k+1) \\
& +\left(2^{h}(h+2 k-2)+1\right) \rho(k) \\
& =\left(3^{h-2}\left((h+3 k)^{2}-7 h-27 k+9\right)\right) \rho(k+1)+2^{h}(h+2 k-2)+1 .
\end{aligned}
$$

Direct computations show

$$
\sum_{h=0}^{n-2}\left(\begin{array}{l}
n \\
h
\end{array}\right)\left(2^{h}(h+2(n-h)-2)+1\right)=3^{n-1}(4 n-6)-2^{n-1}\left(n^{2}+n-6\right)-n-1
$$

hence the problem is to compute

$$
\sum_{h=0}^{n-2}\left(\begin{array}{l}
n \\
h
\end{array}\right)\left(3^{h-2}\left((h+3(n-h))^{2}-7 h-27(n-h)+9\right)\right) \rho(n-h+1) .
$$

Changing the running variable to $k=n-h$ and simplifying the expression it is

$$
\begin{aligned}
& =\sum_{k=2}^{n}\left(\begin{array}{l}
n \\
k
\end{array}\right)\left(3^{n-k-2}\left((n+2 k)^{2}-20 k-7 n+9\right)\right) \rho(k+1) \\
& =3^{-2} \sum_{k=0}^{n}\left(\begin{array}{l}
n \\
k
\end{array}\right)\left(3^{n-k}\left((n+2 k)^{2}-20 k-7 n+9\right)\right) \rho(k+1)-3^{n-2}\left(n^{2}-7 n+9\right)
\end{aligned}
$$

since for $k=1$ it is $\rho(2)=0$. Now the problem is to compute

$$
t:=\sum_{k=0}^{n}\left(\begin{array}{l}
n \\
k
\end{array}\right)\left(3^{n-k}\left((n+2 k)^{2}-20 k-7 n+9\right)\right) \rho(k+1)
$$

Let us switch back to the variable $h$ :

$$
\begin{aligned}
t & =\sum_{h=0}^{n}\left(\begin{array}{l}
n \\
h
\end{array}\right)\left(3^{h}\left((3 n-2 h)^{2}+7 h-27 n+9\right)\right) \rho(n-h+1) \\
& =\left(9 n^{2}-27 n+9\right) \sum_{h=0}^{n}\left(\begin{array}{l}
n \\
h
\end{array}\right) 3^{h} \rho(n-h+1)+(20-12 n) \sum_{h=0}^{n}\left(\begin{array}{l}
n \\
h
\end{array}\right) 3^{h} h \rho(n-h+1) \\
& +4 \sum_{h=0}^{n}\left(\begin{array}{l}
n \\
h
\end{array}\right) 3^{h} h^{2} \rho(n-h+1) .
\end{aligned}
$$

Here comes a trick: set $s:=\sum_{h=0}^{n}\left(\begin{array}{l}n \\ h\end{array}\right) 3^{h}$ (the full sum) and assume $n$ is even; then just the summands corresponding to even $h$ 's contribute in $\sum_{h=0}^{n}\left(\begin{array}{l}n \\ h\end{array}\right) 3^{h} \rho(n-h+1)$. On the other hand, setting

$$
p:=\sum_{\substack{h=0 \\
h \text { even }}}^{n}\left(\begin{array}{l}
n \\
h
\end{array}\right) 3^{h}, d:=\sum_{\substack{h=0 \\
h \text { odd }}}^{n}\left(\begin{array}{l}
n \\
h
\end{array}\right) 3^{h}
$$

it clearly is $s=p+d$. Since $s=(1+3)^{n}=4^{n}=p+d$ and $(1-3)^{n}=2^{n}=p-d$, it follows

$$
\sum_{h=0}^{n}\left(\begin{array}{l}
n \\
h
\end{array}\right) 3^{h} \rho(n-h+1)=p=\frac{1}{2}\left(4^{n}+2^{n}\right) .
$$


If instead $n$ is odd, just the summands corresponding to odd $h$ 's contribute, but this time $(1-3)^{n}=-2^{n}=p-d$ and

$$
\sum_{h=0}^{n}\left(\begin{array}{l}
n \\
h
\end{array}\right) 3^{h} \rho(n-h+1)=d=\frac{1}{2}\left(4^{n}+2^{n}\right),
$$

the same number as before. So, in the end, whatever the parity of $n$ is we get

$$
\sum_{h=0}^{n}\left(\begin{array}{l}
n \\
h
\end{array}\right) 3^{h} \rho(n-h+1)=\frac{1}{2}\left(4^{n}+2^{n}\right) .
$$

Very similar tricks work for the other sums, so we get

$$
\begin{aligned}
& \sum_{h=0}^{n}\left(\begin{array}{l}
n \\
h
\end{array}\right) 3^{h} h \rho(n-h+1)=\frac{3 n}{2}\left(4^{n-1}+2^{n-1}\right) \\
& \sum_{h=0}^{n}\left(\begin{array}{l}
n \\
h
\end{array}\right) 3^{h} h^{2} \rho(n-h+1)=\frac{3 n}{2}\left(4^{n-2}(3(n-1)+4)+2^{n-2}(3(n-1)+2)\right)
\end{aligned}
$$

Now just collect the pieces (and add some other computations) to get the statement.

Remark 28. Comparing the sequences $\left(c_{n}^{\mathbb{Z}_{2}}(A)\right)$ and $\left(c_{n}^{\mathbb{Z}_{2}}(B)\right)$ we may see clearly that both algebras have superexponent 4 , and just the same we see that the rate of growth of $B$ is (asymptotically) half the rate of $A$; yet, it is impressive how similar the two sequences are.

\section{Cocharacter sequences of $A$ And $B$}

We start recalling a generalization of ordinary character convolution.

Definition 29. For all $m, n \in \mathbb{N}$ let $\alpha_{m, n}, \beta_{m, n}$ be assigned $S_{m} \times S_{n}$-characters, and consider the character sequences $\alpha=\left(\alpha_{m, n}\right)_{m, n \in \mathbb{N}}$ and $\beta=\left(\beta_{m, n}\right)_{m, n \in \mathbb{N}}$. The convolution of $\alpha$ and $\beta$ is the character sequence $\alpha \circ \beta$ whose $(m, n)$-element is

$$
(\alpha \circ \beta)_{m, n}:=\sum_{h=0}^{m} \sum_{k=0}^{n}\left(\alpha_{h, k} \otimes \beta_{m-h, n-k}\right)^{S_{m} \times S_{n}} .
$$

We shall simply denote $\alpha \hat{\otimes} \beta$ the induced characters, from now on. An important instance involving the convolution is the relation between the proper cocharacter sequence $\xi(R)=\left(\xi_{m, n}(R)\right)_{m, n \in \mathbb{N}}$ of a superalgebra $R$ and the character sequence $\chi(R)=\left(\chi_{m, n}(R)\right)_{m, n \in \mathbb{N}}$. Indeed, setting up $\alpha_{m, n}:=\delta_{n, 0}(m) \otimes(n)$ (the Kronecker delta) for all $m, n \in \mathbb{N}$, the relation can be expressed simply writing

$$
\chi(R)=\alpha \circ \xi(R) .
$$

Actually, $\alpha$ is the cocharacter sequence of the simple superalgebra $F$ endowed with the trivial $\mathbb{Z}_{2}$-grading. The other natural and simplest character sequence is the one defined by $\beta_{m, n}=(m) \otimes(n)$, which is the cocharacter sequence of the simple superalgebra $D \cong F \oplus \mathbf{t} F$ endowed with its natural grading.

The character sequences $\chi$ obtained as $\chi=\alpha \circ \xi$ are called Young-derived; this because the irreducible characters decomposing $\chi$ follow from the irreducible characters decomposing $\xi$ according to the Young-Pieri rule. Recall that if $\xi_{m, n}=$ $\sum_{\lambda, \mu} c_{\lambda, \mu} \lambda \otimes \mu$ and $\chi_{m, n}=\sum_{\lambda^{\prime}, \mu} c_{\lambda^{\prime}, \mu}^{\prime} \lambda^{\prime} \otimes \mu$ are the decompositions of $\xi$ and $\chi$ 
then $c_{\lambda^{\prime}, \mu}^{\prime}=\sum_{\lambda} c_{\lambda, \mu}$, where $\lambda$ ranges on all partitions $\lambda$ such that $\lambda_{1}^{\prime} \geqslant \lambda_{1} \geqslant \lambda_{2}^{\prime} \geqslant$ $\lambda_{2} \geqslant \lambda_{3}^{\prime} \geqslant \lambda_{3} \geqslant \ldots$

It is easy to get the cocharacter sequence $\chi_{m, n}(B)$ for odd $n$ 's:

Theorem 30. The decomposition of $\chi_{m, 1}(B)=\sum_{\lambda \vdash m} c_{\lambda} \lambda \otimes(1)$ is summarized in the following multiplicity table

\begin{tabular}{|c|c|l|}
\hline$\lambda$ & $c_{\lambda}$ & condition \\
\hline \hline$(m)$ & $m+1$ & \\
$(a, b)$ & $3(a+1-b)$ & $b \geqslant 1$ \\
$(a, b, 1)$ & $2(a+1-b)$ & \\
\hline
\end{tabular}

and the decomposition of $\chi_{m, n}(B)=\sum_{\substack{\lambda \vdash m \\ \mu \vdash n}} c_{\lambda, \mu} \lambda \otimes \mu$ for odd $n \geqslant 3$ is summarized in the following multiplicity table

\begin{tabular}{|c||c|c|c|}
\hline$\lambda \downarrow \mid \mu \rightarrow$ & $(n)$ & $(n-1,1)$ & condition \\
\hline \hline$(m)$ & $2 m+1$ & $2(m+1)$ & \\
$(a, b)$ & $4(a+1-b)$ & $2(a+1-b)$ & $b \geqslant 1$ \\
$(a, b, 1)$ & $2(a+1-b)$ & 0 & \\
\hline
\end{tabular}

Proof. Recall $\xi_{m, 1}(B): \xi_{0,1}=\varnothing \otimes(1), \xi_{1,1}=(1) \otimes(1)$ and $\xi_{m, 1}=((m) \oplus 2(m-$ $1,1)) \otimes(1)$ if $m \geqslant 2$. Then $\chi_{0,1}(B), \chi_{1,1}(B)$ are immediate and for $m \geqslant 2$ we have $\chi_{m, 1}(B)=\sum_{i=0}^{m} \sum_{j=0}^{1} \alpha_{m-i, 1-j} \hat{\otimes} \xi_{i, 1}=\sum_{i=0}^{m} \alpha_{m-i, 0} \hat{\otimes} \xi_{i, 1}=\left((\hat{\alpha} \circ \hat{\alpha})_{m}+2(\hat{\alpha} \circ \eta)_{m}\right) \otimes(1)$,

where $\hat{\alpha}$ is the character sequence $\hat{\alpha}_{h}=(h)$ and $\eta$ is the character sequence defined by $\eta_{m}=(m-1,1)$ if $m \geqslant 2$ and $\eta_{0}=\eta_{1}=0$. The convolution $\hat{\gamma}:=\hat{\alpha} \circ \hat{\alpha}$ is a basic one: the only irreducible characters involved in its decomposition are $(a, b)$ with multiplicity $a+1-b$, with $a+b=m$ and $b \geqslant 0$. We separate the cases $b=0$, that is the character $(m)$ with multiplicity $m+1$, from the cases $b \geqslant 1$. The decomposition of $\zeta:=\hat{\alpha} \circ \eta$ involves just the characters $(a, b)$ and $(a, b, 1)$ (for $b \geqslant 1$ ), both with multiplicity $a+1-b$. Now the result follows.

If we want to compare the cocharacter sequences $\chi_{m, n}(A)$ and $\chi_{m, n}(B)$ we clearly just need to compute $\chi_{m, n}(A)$ for odd $n$ 's, since for even $n$ 's the cocharacters are equal. By the way, the amount of work needed to compute $\chi_{m, n}(A)$ for odd values of $n$ is the same needed for the general case. In principle, it could be recovered from the proper cocharacters $\xi_{m, n}(A)$, as well. In practice, this would be unfair, since the structure of $\Gamma_{m, n}(A)$ is far more complex than $\Gamma_{m, n}(B)$ for odd $n$ 's. There is another approach to get the result, due to the factorability of $T_{2}(A)$. We recall

Theorem 31. (Theorem 6.2 [6])

Let $I, J \unlhd_{2} F\langle Y, Z\rangle$. The cocharacter sequence of $I J$ can be deduced by the cocharacter sequences of $I$ and $J$ according to

$$
\begin{aligned}
\chi_{m, n}(I J) & =\chi_{m, n}(I)+\chi_{m, n}(J)+((1) \otimes \varnothing) \hat{\otimes}(\chi(I) \circ \chi(J))_{m-1, n} \\
& +(\varnothing \otimes(1)) \hat{\otimes}(\chi(I) \circ \chi(J))_{m, n-1}-(\chi(I) \circ \chi(J))_{m, n} .
\end{aligned}
$$

Since $T_{2}(A)=T_{2}(F) T_{2}(D) T_{2}(F)$, we just have to apply twice this last result. Indeed, we already know the cocharacter sequence of $T_{2}(F)$ and $T_{2}(D)$, which we 
called $\alpha$ and $\beta$ respectively. The decompositions will be synthetically displayed through multiplicity tables, reporting partitions, multiplicities and conditions.

Lemma 32. The cocharacter sequence of $T_{2}(F) T_{2}(D)$ is

$$
\chi_{0,0}=1
$$

$\chi_{m, 0}=\chi_{m}^{(0)} \otimes \varnothing$

$\chi_{0,1}=\varnothing \otimes(1)$

$\chi_{m, 1}=\chi_{m}^{(1)} \otimes(1)$

$\chi_{0, n}=\varnothing \otimes(n)+\varnothing \otimes(n-1,1)$

Finally, for $m \geqslant 1$ and $n \geqslant 2$, and, for $m \geqslant 1$,

$$
\chi_{m}^{(0)}:=\begin{array}{r|l|l|}
\hline(m) & 1 & \\
(a, b) & a+1-b & (b \geqslant 1) \\
(a, b, 1) & a+1-b & \\
\hline
\end{array}
$$

and, for $m \geqslant 1$,

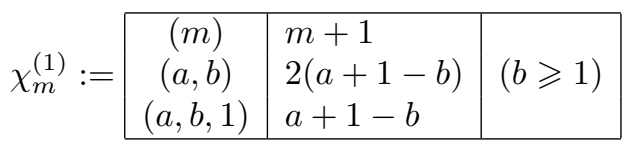

if $n \geqslant 2$.

$\chi_{m, n}=\chi_{m}^{(1)} \otimes(n)+\hat{\gamma}_{m} \otimes(n-1,1)$

$=$\begin{tabular}{|c|c|c|l|}
\hline & $(n)$ & $(n-1,1)$ & \\
\hline$(m)$ & $m+1$ & $m+1$ & \\
$(a, b)$ & $2(a+1-b)$ & $a+1-b$ & $(b \geqslant 1)$ \\
$(a, b, 1)$ & $a+1-b$ & 0 & \\
\hline
\end{tabular}

Proof. The key step is the compute the first convolution $\gamma:=\alpha \circ \beta$, whose generic element $\gamma_{m, n}$ has an easy decomposition: the only couple of partitions occurring in it are $(a, b) \otimes(n)$ for $a+b=m, b \geqslant 0$, with multiplicity $a+1-b$ (the case $b=0$ also follows from the general formula). Hence we may write $\gamma=\hat{\gamma} \otimes \hat{\alpha}$, with abuse of notation. Then just direct computations applying the Young-derived relation are needed.

Now in order to compute the $\chi(A)$ sequence through the relation (1) it is necessary to compute the second convolution $\psi:=\alpha \circ \chi$. Since the structure of characters $\chi_{m, 0}, \chi_{m, 1}$ and $\chi_{m, n}$ with $n \geqslant 2$ changes according to $n$, three cases have to be considered. Patient computational work provides the decomposition of $\psi$ :

Lemma 33. The decomposition of $\psi_{m, n}$ is summarized in the following multiplicity tables:

- If $n=0$ then $\psi_{m, 0}=: \psi_{m}^{(0)} \otimes \varnothing$, where

$$
\psi_{m}^{(0)}=\begin{array}{|c|l|l|}
\hline(a, b) & \frac{1}{2}(a+1-b)(a b+b+2) & b \geqslant 0 \\
(a, b, c) & (a+1-b)(a+2-c)(b+1-c) & c \geqslant 1 \\
(a, b, c, 1) & \frac{1}{2}(a+1-b)(a+2-c)(b+1-c) & \\
\hline
\end{array}
$$

In particular, the multiplicity of $(m)$ in $\psi_{m}^{(0)}$ is $m+1$;

- If $n=1$ then $\psi_{m, n}=: \psi_{m}^{(1)} \otimes(1)$, where La decomposizione di

$$
\psi_{m}^{(1)}=\begin{array}{|c|l|l|}
\hline(m) & \frac{1}{2}(m+1)(m+2) & \\
(a, b) & \frac{1}{2}(a+1-b)(2 a b+a+3 b+2) & (b \geqslant 1) \\
(a, b, c) & \frac{3}{2}(a+1-b)(a+2-c)(b+1-c) & (c \geqslant 1) \\
(a, b, c, 1) & \frac{1}{2}(a+1-b)(a+2-c)(b+1-c) & \\
\hline
\end{array}
$$


- if $n \geqslant 2$ then $\psi_{m, n}=: \psi_{m}^{(1)} \otimes(n)+\psi_{m}^{(2)} \otimes(n-1,1)$, where $\psi_{m}^{(2)}$ is the direct sum of the irreducible $S_{m}$-characters corresponding to the partitions $(a, b, c)$ with multiplicity $\frac{1}{2}(a+1-b)(a+2-c)(b+1-c)$, for all $a \geqslant b \geqslant c \geqslant 0$. In particular,

- the multiplicity of $(a, b)$ is $\frac{1}{2}(a+1-b)(a+2)(b+1)$ (if $\left.c=0\right)$ and

- the multiplicity of $(m)$ is $\frac{1}{2}(m+1)(m+2)$ (if $\left.b=c=0\right)$.

Now relation (1) provides the cocharacter sequence $\chi(A)$, by direct computations. By the way, not just the cases $m=0$ or $m>0$ make difference in the formula, but also the cases $n=0,1,2,3$ and $n \geqslant 4$ (because the character $\psi_{h, k}$ changes its structure in $(\varnothing \otimes(1)) \hat{\otimes} \psi_{m, n-1}$.

Here we just give the decompositions of the characters $\chi_{m, 0}(A)$ and the characters $\chi_{m, 1}(A)$ :

Proposition 34. It is $\chi_{0,0}(A)=1$ and, for $m \geqslant 1, \chi_{m, 0}(A)=: \hat{\chi}_{m}^{(0)} \otimes \varnothing$, where

$$
\hat{\chi}_{m}^{(0)}=\begin{array}{r|l|l|}
(m) & 1 & b \geqslant 1 \\
(a, b) & \frac{1}{2}(a+1-b)(a b-a+2) & \\
(a, b, 1) & \frac{1}{2}(a+1-b)(3 a b-2 a+b) & \\
(a, b, c) & 2(a+1-b)(a+2-c)(b+1-c) & c \geqslant 2 \\
(a, b, 1,1) & \frac{1}{2}(a+1-b)(3 a b-a+2 b-2) & \\
(a, b, c, 1) & 2(a+1-b)(a+2-c)(b+1-c) & c \geqslant 2 \\
(a, b, c, 2) & \frac{1}{2}(a+1-b)(a+2-c)(b+1-c) & c \geqslant 2 \\
(a, b, c, 1,1) & \frac{1}{2}(a+1-b)(a+2-c)(b+1-c) & \\
\hline
\end{array}
$$

Of course this also is the cocharacter sequence $\left(\chi_{m, 0}(B)\right)_{m \in \mathbb{N}}$.

Remark 35. The sequence $\chi_{m, 0}(A)$ is essentially the sequence $\hat{\chi}_{m}^{(0)}$. It is easy to see that $\hat{\chi}$ is actually an important cocharacter sequence, and precisely it is the cocharacter sequence of the (non-graded) algebra $U T_{3}(F)$. The proper cocharacter sequence was obtained in [16], and from its description it is possible to obtain $\hat{\chi}$. By the way, in order to get the decomposition of $\xi\left(U T_{3}(F)\right)$ the more general Littlewood-Richardson rule is needed, so a direct approach could be quite hard. To the best of our knowledge, the explicit cocharacter sequence of $U T_{3}(F)$ has not been published so far, so we record it here, as a byproduct of our subject.

In the same spirit, notice that $\chi^{(0)}$ is actually $\chi\left(U T_{2}(F)\right)$ and, of course, the original version of formula 1 (see [25]) can be used for all $U T_{n}(F)$.

Proposition 36. It is $\chi_{0,1}(A)=\varnothing \otimes(1)$ and, for $m \geqslant 1, \chi_{m, 1}(A)=: \hat{\chi}_{m}^{(1)} \otimes(1)$, where

$$
\hat{\chi}_{m}^{(1)}=\chi_{m}^{(1)}+(1) \hat{\otimes} \psi_{m-1}^{(1)}+\psi_{m}^{(0)}-\psi_{m}^{(1)}
$$


has the following decomposition into irreducible characters

$$
\hat{\chi}_{m}^{(1)}=\begin{array}{r|l|l|}
(m) & m+1 & b \geqslant 1 \\
(a, b) & \frac{1}{2}(a+1-b)(3 a b-a+2 b+2) & \\
(a, b, 1) & \frac{1}{2}(a+1-b)(7 a b-2 a+5 b-2) & \\
(a, b, c) & 4(a+1-b)(a+2-c)(b+1-c) & c \geqslant 2 \\
(a, b, 1,1) & \frac{1}{2}(a+1-b)(5 a b-a+4 b-2) & \\
(a, b, c, 1) & 3(a+1-b)(a+2-c)(b+1-c) & c \geqslant 2 \\
(a, b, c, 2) & \frac{1}{2}(a+1-b)(a+2-c)(b+1-c) & \\
(a, b, c, 1,1) & \frac{1}{2}(a+1-b)(a+2-c)(b+1-c) & \\
\hline
\end{array}
$$

We included this sequence to compare at least the easiest case in which $\chi(B)$ and $\chi(A)$ differ. On the other hand, here all partitions $\lambda \vdash m$ occurring in the decomposition of the general cases appear. In the other cases, the $Z$-components will differ from the simple (1), and precisely in the irreducible character $\lambda \otimes \mu$ the partitions $\mu \vdash n$ will be the following: $(n),(n-1,1),(n-2,2),(n-2,1,1)$. Then the multiplicities will change according the cases $m=0$ or $m \geqslant 1$ and $n=0,1,2,3$ or $n \geqslant 3$, so one may get an idea of what the decompositions are, apart from the precise multiplicities. By the way, we computed all of them, so they are available upon requesting the corresponding author.

\section{REFERENCES}

[1] E. Aljadeff, A. Giambruno, Multialternating graded polynomials and growth of polynomial identities, Proc. Amer. Math. Soc. 141 (2013), 3055-3065.

[2] E. Aljadeff, A. Giambruno, D. La Mattina, Graded polynomial identities and exponential growth, J. Reine Angew. Math. 650 (2011), 83 - 100.

[3] A. Berele, Cocharacters of Z/2Z-graded algebras, Isr. J. Math. 61 (1988), 225 - 234.

[4] F. Benanti, A. Giambruno, M. Pipitone, Polynomial identities on superalgebras and exponential growth, J. Algebra 269 (2003), 422-438.

[5] O.M. Di Vincenzo, On the graded identities of $M_{1,1}(E)$, Isr. J. Math. 80 (3) (1992), 323 335.

[6] O. M. Di Vincenzo, R. La Scala, Block-triangular matrix algebras and factorable ideals of graded polynomial identities, J. Algebra 279 (2004), 260-279.

[7] O. M. Di Vincenzo, R. La Scala, Minimal algebras with respect to their *-exponent, J. Algebra 317 (2007), 642-657.

[8] O. M. Di Vincenzo, V. Nardozza, On the *-polynomial identities of a class of $*$-minimal algebras, Comm. Algebra 38 (2010), 3078-3093.

[9] O. M. Di Vincenzo, E. Spinelli A characterization of $*$-minimal algebras with involution, Isr. J. Math. 186 (2011), 381-400.

[10] O. M. Di Vincenzo, E. Spinelli, On some minimal supervarieties of exponential growth, J. Algebra 368 (2012), 182-198.

[11] O. M. Di Vincenzo, E. Spinelli, Minimal varieties of associative PI (super)-algebras with respect to their (graded) exponent, São Paulo Journal of Mathematical Sciences, 10 (2) (2016), 248-262.

[12] V. Drensky, Codimensions of T-ideals and Hilbert series of relatively free algebras, J. Algebra 91 (1984), 1-17.

[13] V. Drensky, Extremal varieties of algebras I, Serdica 13 (1987), 320-332 (Russian).

[14] V. Drensky, Extremal varieties of algebras II, Serdica 14 (1988), 20-27 (Russian).

[15] V. Drensky, A. Giambruno, Cocharacters, codimensions and Hilbert series of the polynomial identities for $2 \times 2$ matrices with involution, Canad. J. Math. 46 (1994), 718-733.

[16] V. Drensky, A. Kasparian, Polynomial identities of eighth degree for $3 \times 3$ matrices, Annuarire de l'Univ. de Sofia, Fac. de Math et Meccan., Livre 1, Math. 77, 175-195. 
[17] O. M. Di Vincenzo, V. Drensky, V. Nardozza, Subvarieties of the Varieties Generated by the Superalgebra $M_{1,1}(E)$ or $M_{2}(K)$, Comm. Algebra 31 (1), (2003)

[18] A. Giambruno, D. La Mattina, Graded polynomial identities and codimensions: Computing the exponential growth, Adv. Math. 225 (2010), 859-881.

[19] A. Giambruno, M. Zaicev, On codimension growth of finitely generated associative algebras, Adv. Math. 140 (1998), 145-155.

[20] A. Giambruno, M. Zaicev, Exponential codimension growth of P.I. algebras: and exact estimate, Adv. Math. 142 (1999), 221-243.

[21] A. Giambruno, M. Zaicev, Involutions codimensions of finite dimensional algebras and exponential growth, J. Algebra 222 (1999), 471-484.

[22] A. Giabruno, M. Zaicev, Codimension growth and minimal superalgebras, Trans. Amer. Math. Soc. 355 (2003), 5091-5117.

[23] A. R. Kemer, Varieties and $\mathbb{Z}_{2}$-graded algebras, Izv. Akad. Nauk SSSR, Ser. Mat. 48 (1984), 1042-1059. Translation: Math. USSR Izv. 25 (1985), 359-374

[24] A. Regev, Existence of identities in $A \otimes B$, Israel J. Math. 11 (1972), 131-152.

[25] A. Regev, Young-derived sequences of $S_{n}$-characters, Adv. Math. 106 (1994), 169-197.

Dipartimento di Matematica, Informatica ed Economia, Università degli Studi della

Basilicata, viale dell'Ateneo Lucano 10, 85100 Potenza, Italia

Email address: onofrio.divincenzo@unibas.it

Dipartimento di Matematica, Università degli Studi di Bari, via Orabona 4, 70125 BARI, ITALIA

Email address: vincenzo.nardozza@uniba.it 